\title{
Histomorphometric evaluation of critical-sized bone defects using Osteomeasure and Aperio image analysis systems.
}

\author{
Flavia Medeiros Savi, BSc, ${ }^{1}$ Felicity Lawrence, BSc, ${ }^{1}$ Dietmar Werner Hutmacher, $\mathrm{PhD},{ }^{1,2}$ \\ Maria Ann Woodruff, PhD, ${ }^{1,2,3}$ Laura Jane Bray ${ }^{*}, \mathrm{PhD}^{1}{ }^{1}$ Marie-Luise Wille, $\mathrm{PhD}^{{ }^{*}}$ \\ * These authors share senior authorship \\ 1. Institute of Health and Biomedical Innovation, Queensland University of Technology, 60 \\ Musk Avenue, Kelvin Grove, Queensland 4059, Australia. Phone: (61) 31386297 \\ 2. ARC Centre for Additive Biomanufacturing, Queensland University of Technology, Kelvin \\ Grove, Australia \\ 3. Biofabrication and Tissue Morphology Group, Queensland University of Technology, \\ Institute of Health and Biomedical Innovation, Kelvin Grove, Queensland, Australia. \\ Contact details: \\ Flavia Medeiros Savi \\ Institute of Health and Biomedical Innovation, Queensland University of Technology, 60 \\ Musk Avenue, Kelvin Grove, Queensland 4059, Australia. Phone: (+61) 0731386297. \\ Email: f.medeirossavi@hdr.qut.edu.au
}

\section{Felicity Lawrence}

Institute of Health and Biomedical Innovation, Queensland University of Technology, 60 Musk Avenue, Kelvin Grove, Queensland 4059, Australia. Phone: (+61) 0731386247. Email: f.lawrence@qut.edu.au

Distinguished Professor Dietmar Werner Hutmacher

QUT Chair in Regenerative Medicine. Institute of Health and Biomedical Innovation, Queensland University of Technology, 60 Musk Avenue, Kelvin Grove, Queensland 4059, Australia. Phone: (61) 7313 86077, Fax: (+61) 073138 6030. Email: dietmar.hutmacher@qut.edu.au 
Professor Maria Ann Woodruff

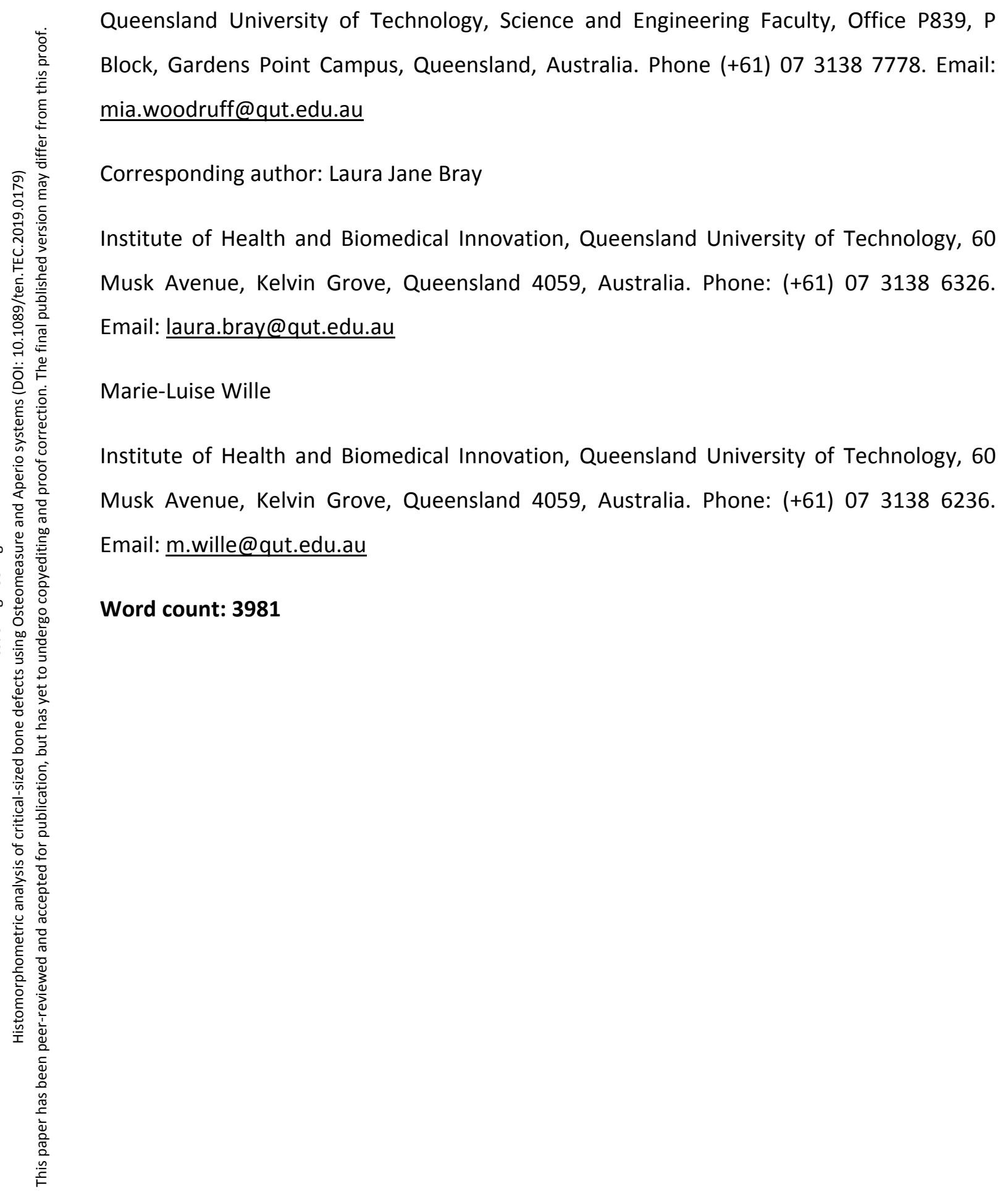




\section{Abstract}

Most histological evaluations of critical-sized bone defects are limited to the analysis of a few regions of interest at a time. Manual and semi-automated histomorphometric approaches often have intra- and inter-observer subjectivity, as well as variability in image analysis methods. Moreover, the production of large image data sets makes histological assessment and histomorphometric analysis labour intensive and time-consuming. Herein, we tested and compared two image segmentation methods: thresholding (automated) and region-based (manual) modes, for quantifying complete image sets across entire criticalsized bone defects, using the widely used Osteomeasure system and the freely downloadable Aperio Image Scope software. A comparison of bone histomorphometric data showed strong agreement between the automated segmentation mode of the Osteomeasure software with the manual segmentation mode of Aperio Image Scope analysis (bone formation $R^{2}=0.9615$ and fibrous tissue formation $R^{2}=0.8734$ ). These results indicate that Aperio is capable of handling large histological images, with excellent speed performance in producing highly consistent histomorphometric evaluations compared to the Osteomeasure image analysis system. The statistical evaluation of these two major bone parameters demonstrated that Aperio Image Scope is as capable as Osteomeasure. This study developed a protocol to improve the quality of results and reduce analysis time, while also promoting the standardization of image analysis protocols for the histomorphometric analysis of critical-sized bone defect samples.

\section{Impact Statement}

Despite bone tissue engineering innovations increasing over the last decade, histomorphometric analysis of large bone defects used to study such approaches continues to pose a challenge for pathological assessment. This is due to the resulting large image data set, and the lack of a gold standard image analysis protocol to quantify histological outcomes. Herein we present a standardized protocol for the image analysis of critical-sized bone defect samples stained with Goldner's trichrome using the Osteomeasure and Aperio Image Scope image analysis systems. The results were critically examined to determine their reproducibility and accuracy for analyzing large bone defects. 


\section{Introduction}

The reconstitution of critical-sized bone defects via tissue engineering approaches has received considerable scientific, economic and clinical interest over the past years ${ }^{1}$ with the use of animal models and histological assessment, making it possible to investigate the pathways that regulate bone healing. ${ }^{2}$ Despite multiple tissue engineering innovations over the last decade, histomorphometric analysis of large bone defects continues to pose a challenge for pathological assessment due to the resulting large image data set, and the lack of a gold standard histomorphometric image analysis software system and approach/protocol to quantify critical-sized bone defect histological outcomes.

For the last fifty years, qualitative assessment of histological sections has been the gold standard approach for evaluating bone responses to bone therapies. ${ }^{3,4}$ Most histological interpretations of critical-sized bone defects are made according to the presence or absence of staining. ${ }^{5,6}$ Qualitative quantification also has been used to interpret histological outcomes. Usually graded according to staining intensity, such quantifications can be categorized as weak $(+)$, medium $(++)$ and strong $(+++){ }^{7}$

When numbers are used instead of qualitative signs, these histological evaluations are termed semi-quantitative estimations and are widely used for assessment of immunohistochemical (IHC) stains as well as biological response evaluations to medical devices. $^{4,7,16,8-15}$ In their 2014 review, Fedchenko \& Reifenrath ${ }^{7}$ described the use of different approaches for the interpretation and reporting of IHC results in bone tissue studies. After extensive research, they were able to identify six predominant methodologies, including: 1 . description of morphological parameters; 2 . counting of positively stained cells; 3 . calculating positive percentages of stained cells in relation to the total area evaluated; 4. qualitative scoring related to staining intensity; 5 . combinative semi-quantitative scoring and 6 . automated systems. However, a standardized system has not yet been agreed on for the quantitative evaluation of staining results.

Over the last decades, the extraction of numerical data from histological images of bone defect samples has gained significant attention with the introduction of whole slide imaging scanners and the integration of statistical methods into medical image analysis. ${ }^{4,17}$ 
The increase in computer power and the improvement in integrated image analysis systems have automatically enabled histological analysis to move from purely qualitative analysis to semi-automated and automated quantitative measurements. ${ }^{18,19}$ Histomorphometric analysis rather than computer-assisted image analysis innovations has been considered the gold standard methodology for bone quantitative evaluations. ${ }^{4}$

Despite the development of new imaging technologies and the advantage of entire microscope glass slides being imaged, histomorphometric evaluations of critical-sized bone defects present numerous intrinsic and extrinsic challenges including the length of the defect $(30 \mathrm{~mm}$ defect plus $\sim 10 \mathrm{~mm}$ distal and $10 \mathrm{~mm}$ proximal host bone $=\sim 50 \mathrm{~mm}$ total defect size), which produces large histological image files. The file size of a high-resolution image can easily reach 20 gigabytes (for $1.29 \mu \mathrm{m}$ resolution - 5X microscope objective, 39904 x 19909 pixels image file).

To date, considerations such as the length of the bone defect and image file size ensure most of the histomorphometric evaluations of critical-sized bone defects remain limited to the analysis of a few regions of interest (ROI) at a time. Moreover, semi-automated methods (manual histomorphometric assessment) have intra- and inter-observer variability, ${ }^{20}$ primary data cannot be reanalysed automatically as the histological image is not retained with the biopsy results file, ${ }^{4}$ and the appraisal of large image data sets makes histological assessment and histomorphometric analysis labour intensive and timeconsuming.

The large volume of data obtained from the imaging of critical-sized bone defect samples also raises a number of economical, technical and methodological challenges, including: high costs; the acquisition, projection, viewing, storage, and analysis of content-rich images; lack of interoperability between image acquisition format and image analysis systems; poor image analysis user interface; inability to analyse multiple parameters (variables) in parallel; and no delivery of information in a systematic manner. Moreover, none of the technical factors mentioned have been standardized. ${ }^{21}$

Histomorphometry is a quantitative approach used for the extraction of numerical data through the measurement of tissue samples using digitized images. ${ }^{17,22-24}$ The 
recommended histomorphometric nomenclature, symbols and units as well as appropriate approaches and methods relevant to bone indices' calculations have been described by Michael Parfitt ${ }^{25}$ and David Dempster. ${ }^{23}$ However, this standardization refers to bone histomorphometry terminology and dimensional extrapolation via the application of stereology, rather than a gold standard histomorphometric image analysis system/tool. ${ }^{25}$

In summary, the Osteomeasure image analysis system is frequently used for obtaining histomorphometric evaluations from dynamic or digitized images. ${ }^{26-29}$ It is often referred to as the gold standard. Primary (2D measurements) and derived measurements (3D measurements) can be obtained, however experience and knowledge are required for its use. In contrast, the Aperio Image Scope image analysis system is freely available, requires little technical expertise, and it has short processing times. Hence, this software has potential for expediting the histomorphometric evaluation of critical-sized bone defect image data sets, and should be explored further. Herein we present a standardized protocol for the image analysis of critical-sized bone defect samples stained with Goldner's trichrome using the Osteomeasure and Aperio Image Scope image analysis systems using manual and automated image segmentation modes.

\section{Material and methods}

Histology

Histological resin sections of sheep tibiae collected from seventeen Merino sheep were sourced from a previous study (Queensland University of Technology Animal Ethics Approval Number 0900000425). Three different treatment groups, each containing samples with a critical sized $3 \mathrm{~cm}$ tibial defect, were evaluated in this study: group I $(n=3)$, a microfiber mesh-scaffold only; group II $(n=5)$, the microfiber mesh-scaffold was combined with functionalized alginate hydrogel and group III ( $n=9$ ), functionalized alginate hydrogel combined with recombinant human bone morphogenic protein 2 (rhBMP-2) (see supplemental material 1 for more detail about the treatment groups).

Histological assessment was performed on ground resin sections stained with Goldner's trichrome staining (histological samples preparation is described in supplemental material 1). 
Resin ground sections were entirely imaged using ZEN software on a Carl Zeiss microscope (Carls Zeiss Microscopy, NY, USA, ZEISS Axio Imager 2) (czi format images) with a $2.5 \mathrm{X}$ objective and $2.58 \mu \mathrm{m}$ per pixel resolution (also known as the scaling factor) (see supplemental material 2 for further details about image acquisition). Images were exported in TIFF format to be used in the Osteomeasure system and in Big TIFF pyramid format to be used in the Aperio Image Scope system. A sub-set of images limited to the defect site only ROI was defined using the Aperio Image Scope image analysis system. The defect perimeter of $30 \mathrm{~mm}$ was defined excluding proximal and distal host bone. (See supplemental material 2 for further details about image properties).

Histomorphometry

Seventeen bone defect only ROI images were analysed with the Osteomeasure and Aperio Image Scope analysis systems. Quantification of staining patterns for the formation of new bone and fibrous tissue formation was performed using both the manual delineation of tissue edges (manual - region based selection mode) and the automation of colour-specific threshold ranges (automated - thresholding segmentation mode).

Using the Osteomeasure and Aperio Image Scope systems, the total tissue area of the defect only ROI was manually traced. Then, manual and automated segmentation modes were used to acquire histomorphometric indices for two major bone variables including: bone and soft tissue. A protocol for the Osteomeasure and Aperio image scope systems for histomorphometric evaluation of static images using the manual and automated segmentation modes can be found in supplemental material in Supplemental material 3 and 4 , respectively.

The following criteria were used when measuring the major bone variables using the Osteomeasure and Aperio Image Scope systems:

1. Total tissue area: The total area of all stained tissue structures within the $30 \mathrm{~mm}$ defect only ROI;

2. Bone area: tones of green and blue color produced by the Goldner's trichrome stain within the $30 \mathrm{~mm}$ defect only ROI; 
3. Fibrous tissue area: tones of red color produced by the Goldner's trichrome stain within the $30 \mathrm{~mm}$ defect only ROI.

4. All manual and automated area selections must be acquired using the same software system magnification (2X) (Osteomeasure system only).

Primary measurements acquired according to the nomenclature and indices' calculations describe in Parfitt et $a l^{25}$ included:

1. Total Tissue Area (T. Ar): amount of the total tissue area within the defect only ROI; acquired through manual area selection only.

2. Bone Area (B. Ar): amount of the total area of bone tissue within the defect only ROI; acquired through manual and automated area selections.

3. Fibrous Tissue area (Fb. Ar): amount of total fibrous tissue area within the defect only ROI; acquired through manual and automated area selections.

Statistical analysis

Statistical analysis was obtained with Graph Pad Prism version 7.03 and excel. Intraobserver reproducibility (concordance of measurements done by one observer) and agreement between the image systems' results were evaluated by correlation coefficient $\left(R^{2}\right)$ with $95 \%$ confidence intervals $(\mathrm{Cl})$. A correlation coefficient of 1 or -1 corresponded to a perfect linear correlation between two measurements. A 0 value corresponded to no correlation between measurements. Inter system Bland-Altman analysis was performed between image analysis segmentation methods. Mean value, standard deviation (SD) and standard error of the mean (SEM) were compared between the two image analysis systems according to segmentation modes, variables and per treatment group. One-way Anova was used to compare treatment groups and $t$-test was used compare image analysis system (Aperio vs Osteomeasure) measurements of bone and fibrous tissue formation per group treatment. Significance was defined as $p<0.05$.

\section{Results}

Using the Osteomeasure software, it took approximately 30 minutes to analyse each image in manual mode and 1 hour when using the automated (thresholding) segmentation 
mode. Using Aperio software, it took approximately 20 minutes and 10 minutes to analyse the same images using the manual and automated segmentation modes respectively.

The results obtained for bone and fibrous tissue formation within the defect only ROI were analysed without discrimination of treatment groups to determine inter-system (Osteomeasure vs. Aperio) parity of measurements.

Inter system comparisons for the bone and fibrous tissue variables using different segmentation modes

The automated bone and fibrous tissue paired measurements obtained with the Aperio and Osteomeasure systems presented both high and positive correlation (Bone: $\mathrm{R}^{2}=$ $0.8754,95 \% \mathrm{Cl}: 0.8268$ to 0.9769 and Fibrous tissue: $\mathrm{R}^{2}=0.8464,95 \% \mathrm{Cl}: 0.7877$ to 0.9712). Although, high and positive correlation was found, the Bland-Altman paired analysis indicate that the bone (Fig. 1 A) and fibrous tissue formation (Fig. 1 B) measurements obtained with the Aperio Image Scope were mostly higher than the measurements obtained with the Osteomeasure system, suggesting a small variability within the difference of the Aperio and Osteomeasure automated paired measurements. However, most of the bone and fibrous tissue paired measurements were lying within the 95\% limits of agreement, except for one of the bone paired measurements (Fig. 1 A).

The manual bone paired measurements attained with the Aperio Image Scope system were in excellent agreement with the Osteomeasure system measurements $\left(R^{2}=0.9117\right.$, $95 \% \mathrm{Cl}: 0.8764$ to 0.9839$)$. Most of the paired measurement were within $95 \%$ limits of agreement of the Bland-Altman analysis, except for one pair of measurements, in which the Aperio histomorphometric measurement was lower than the evaluation attained with the Osteomeasure system (Fig. 1 C).The manual fibrous tissue measurements attained with the Aperio system was positively correlated with the Osteomeasure manual results $\left(R^{2}=0.8734,95 \% \mathrm{Cl}: 0.8241\right.$ to 0.9765$)$. Although the manual correlation was higher than the correlation obtained with the automated segmentation mode, one pair of measurements was lying outside the Bland-Altman's 95\% limits of agreement (Fig. 1 D).

Figure 2 illustrates an overview and comparison between Osteomeasure and Aperio Image Scope systems when using thresholding (automated) and manual (region-based selection) 
segmentation modes. The images show that when using the automated segmentation mode, both Osteomeasure and Aperio Image Scope systems produced a finer image segmentation than with the manual segmentation mode (Fig. 2 B - E). However, higher bone measurements and a discrepant paired measurement were found for the bone formation variable using the automated segmentation of the Aperio system.

When examining the data (supplementary material 5) to identify the outliers obtained in the inter system comparisons, these results were determined to be the result of nonspecific background. In this study, bone resin images suffered from non-specific background staining as a result of resin surface etching prior to Goldner's trichrome staining. Contrary to paraffin samples, where paraffin is completely removed prior to staining, it is not possible to remove resin from the resin slides. Samples are etched in order to deliberately crack the resin allowing stain penetration. This procedure results in resin etching artefacts such as: fissures in the resin (Fig. $2 \mathrm{~F}$ ). These fissures mostly appear in the void areas such as: the resin around the tissue, in the central marrow cavities (Fig 2 F) and within the fibrous tissue areas (Fig. 2 G), ensuing additional stain (mostly the bone variable tones). Manual segmentation mode also affected the bone formation histomorphometric measurements, however while using the Osteomeasure system. The bone marrow area cannot be completely and equally isolated without been affected by user manual selection. As such the outliers obtained for the bone manual segmentation mode using the Osteomeasure system were identified as intra-observer variability error.

The previous inter system comparisons (Fig. 1) showed that the automated segmentation mode of the Osteomeasure system (Fig. 2 B) and the manual segmentation mode of the Aperio Image Scope (Fig. 2 E) system produced a finer image pixel segmentation. Thus, in order to compare the different treatment groups, for bone and fibrous tissue formation the automated measurements scores obtained with the Osteomeasure system were plotted against the manual measurements obtained with the Aperio Image Scope system (Fig. $3 \mathrm{~A}$ and $\mathrm{B}$ ). 
In terms of new bone and fibrous tissue formation, strong correlation of $R^{2}=0.9615$ and $R^{2}$ $=0.8734$ was attained, respectively. The corresponding Bland-Altman analysis indicated nearly perfect agreement between the measurements for the bone formation histomorphometric variable (Fig. 3 A).

Histomorphometric results according to treatment groups

To compare the treatment groups ANOVA analysis was applied using the same histomorphometric data set (Fig. 4 A - D).

Significantly higher amounts of bone formation were observed within group III when compared to group I when using the Osteomeasure automated segmentation mode analysis (ANOVA $p=0.0218$ ) and when using the Aperio Image Scope manual segmentation mode analysis (ANOVA $p=0.0247$ ) (Fig. $4 \mathrm{~A}$ and $\mathrm{C}$ ). Correspondingly, $a$ significantly lower amount of fibrous tissue formation was observed between group III and group I using the Osteomeasure automated segmentation mode (ANOVA $p=0.0288$ ) and using the Aperio Image scope manual segmentation mode analysis (ANOVA $p=0.0076$ ) (Fig. $4 \mathrm{~B}$ and D).

Although, the $p$ values found were slightly different, the results attained with the Osteomeasure automated segmentation mode and Aperio Image Scope manual segmentation mode followed comparable trends as illustrated in Figure $5 \mathrm{~A}$ and $\mathrm{B}$.

\section{Descriptive inter systems statistical analysis}

The accuracy of the inter system descriptive statistical analyses was determined by comparing the SD of the Aperio Image Scope image analysis software to the widely used Osteomeasure system. 95\% coefficient intervals were also determined (Table 1). Overall, the descriptive statistical analyses indicated that SD values obtained for the Aperio Image Scope image analysis was slightly lower than the SD obtained with the Osteomeasure system, and thus suggesting comparability in terms of its precision (Table 1 ).

Similarly, inter system descriptive analyses of bone and fibrous tissue formation per group treatment obtained with the Aperio Image Scope also did not differ from the Osteomeasure system, further confirmed by calculated $p$ values $>0.05$ (Table 2). However, 
12

these results denote that the sampling size was small, hence the wide $95 \%$ coefficient intervals. ${ }^{30}$ These results suggest that the accuracy of the measurements obtained with the Aperio Image Scope are as precise as the Osteomeasure system analysis.

\section{Discussion}

The digitalization of microscope slide sections has allowed the capture of histological images to shift from partial image acquisition to scanning whole microscope glass slides, further improving and refining histological image assessments. ${ }^{31}$ The content and quality of histological images are directly associated with the microscope optics used when acquiring the histological image. While the Tagged Image File Format (TIFF) is a widely accepted image format, owing to its capabilities and flexibility, the TIFF images produced by the Zen Software were not supported by the Aperio Image Scope system. Thus, the images had to be saved as Big TIFF/pyramid format, enabling compatibility between image analysis software and the displayed image format.

Image segmentation approaches commonly include: i) thresholding; ii) edge-based; iii) region based and iv) active contour modes. ${ }^{32}$ Our histomorphometric analyses were carried out using two well-known image analysis systems, Osteomeasure and Aperio Image Scope, comparing two image segmentation methods, thresholding and regionbased modes, using Goldner's trichrome stained sections of critical-sized bone defect samples. Variability of stain colours, pixel colour intensity overlap due to heterogeneity of staining colour, as well as image staining background were the main issues associated with the thresholding (automated) image segmentation mode. Lejeune et $a{ }^{20}{ }^{20}$ while validating a new computer-assisted image analysis procedure, described similar findings with stain reproducibility and the necessity for addressing consistent methods for quantification of staining. Within void areas where no tissue structures exist, background staining may occur due to resin etching artefacts. Background pixel intensities that closely mimic the colours produced by the Goldner's trichrome stain (such as the shades of blue and green for the bone variable), also are included in the histomorphometric quantification, and would explain the higher bone formation measurements obtained with the automated segmentation mode while using the Aperio Image Scope analysis. Changing the histogram 
intensity for removing image background is not recommended. This type of operation alters the image content permanently by changing the pixel values. Once it is applied and the image is saved, image data cannot be retrieved unless the sample is imaged again. ${ }^{33}$ This issue also compromised the fibrous tissue automated results obtained with the Aperio image analysis system. In this case, the central cavities (trabeculae spaces) filled with hematopoietic tissue and areas of non-mineralized bone which stained reddish (same colour of fibrous tissue), also were counted as fibrous tissue, and would also explain the higher measurements attained.

A similar issue happens while using the Osteomeasure system, however instead of selecting staining background, the system selects the central marrow area cavities within the bone defect site. This could be related to the Osteomeasure processing steps for the thresholding of pixel acquisition. After thresholding selection, the Osteomeasure system requires smoothing of the thresholding selection followed by acceptance of the pixel selection. During the smoothing step, extra blurring is included in the selection, hence adding extra pixels to the final histomorphometric quantification. Hangartner ${ }^{34}$ reported the influence of thresholding techniques on the selection of bone parameters as a similar blurring artefact. The reason for such an effect relies on the finite resolution of the image matrix. A pixel can be represented only by one value, therefore if bone tissue and marrow cavities are sharing the edges or part of a pixel, the selection will be based on an average of all tissues at that location. This issue was observed while using the automated image segmentation of the Osteomeasure system, where bone marrow cavities within the bone tissue had additional blurring artefact, thus selecting extra pixels within an image.

In line with the colour overlap mentioned previously, the present study observed that manual segmentation mode using the Aperio image Scope system took longer to achieve histomorphometric evaluation than the automated segmentation mode. However, manual selection was still, on average, ten minutes faster than the automated segmentation mode using the Osteomeasure system. Corroborating our finding, Zhang et $a l^{4}$ obtained similar results while using Osteomeasure automated segmentation mode for histomorphometry of whole slide imaging. This large difference in time may be related to the Osteomeasure image analysis software system approach. ${ }^{19}$ Automated segmentation saved time when 
colour overlap was not an issue, however when colour overlapping was problematic, it took some time to obtain the right pixel colour selection.

A solid understanding of image analysis software and image properties is required to generate reliable conclusions. Histomorphometric measurements in digital images can be compromised by several factors, such as image dimensions and scaling factors. Therefore, image analysis system calibration is compulsory to ensure reproducibility and accuracy of histomorphometric measurements. Prior to any measurement taken, it is imperative to retrieve the hierarchical framework (image raw data/image properties) of the image processing parameters. False conclusions can result due to poor knowledge and understanding of image and image analysis software features.

With the Osteomeasure system, when the images are loaded and read by the system, they are manually scaled according to the TIFF image. Then, the user needs to follow a sequence of steps from retrieval of image properties, such as dimensions and scale per pixel, to the conversion of image width and high from pixels to microns. In comparison, Aperio Image Scope is relatively easy to calibrate as the user only needs to insert the resolution per pixel by which the image was acquired.

Aperio Image Scope also offers the advantage of working with the entire image section by using selection tools, such as the rectangle tool selection to select the area of the region of interest (defect site) within an image. However, with regards to interoperability between image file format and image analysis system, disadvantages are encountered including: image dimensions and format restriction.

Another limitation of working with the Aperio Image Scope system is that the user has to find out the pixel value of a specific color to adjust the algorithm selection. Image color pixel information is provided as RGB color mode. With the Aperio Image Scope system, the RGB color mode must be converted to HSI color mode, which extends the time spent to tune the algorithm selection. In addition, image background must be excluded from the analysis, thus manual image segmentation gives a finer histomorphometric evaluation. An additional drawback is that the histomorphometric data generated only provide the total 
tissue area in $\mathrm{mm}^{2}$, while the other specific measurements, such as the variables used in this study, need to be converted from pixels to $\mathrm{mm}^{2}$ afterwards.

The Osteomeasure system also presents a few disadvantages including high costs and inability to analyse multiple parameters in parallel or reanalyse primary data automatically since the histological image is not retained with the analysis results file. It also requires fine hand motor coordination, as manual tracing cannot be interrupted in the middle of a manual tissue area selection. Moreover, manual tracing is a time consuming and labour intensive method, especially while appraising large image data sets. ${ }^{4}$ Such a technique directly depends on the subjective abilities of the user and it is prone to inconsistencies between analyses (intra-observer) and users (inter-observer).

With the increasing use of histomorphometric evaluations, the key aim of this study was to identify whether the histomorphometric data produced by the Aperio Image Scope was sufficiently reliable when compared with the widely used Osteomeasure image analysis system. Comparison of the image analysis systems and segmentation modes was made using the same critical-sized bone defect image data set, and it was found that the results obtained with the Osteomeasure and Aperio Image Scope systems were remarkably similar with regards to the ANOVA analysis obtained for bone formation and slightly different for the fibrous tissue histomorphometric variable. The freely available Aperio Image Scope software permitted both manual and automated segmentation mode approaches, enabled the production of highly similar histomorphometric data, required minimal supervision and user training, and was far more expeditious, interface friendly and cost effective. However, no single image analysis package is perfect for every application. Image analysis systems vary in their functionality and interoperability with regards to different histological sample types and sizes, including the image acquisition software used for obtaining the histological images.

\section{Acknowledgements}

The authors would like to thank to the Histology Facility Central Analytical Research Facility (CARF), Institute of Health and Biomedical Innovation (IHBI) at Queensland University of Technology (QUT) for providing general facility support. 
Page 16 of 45

\section{Author Disclosure Statement}

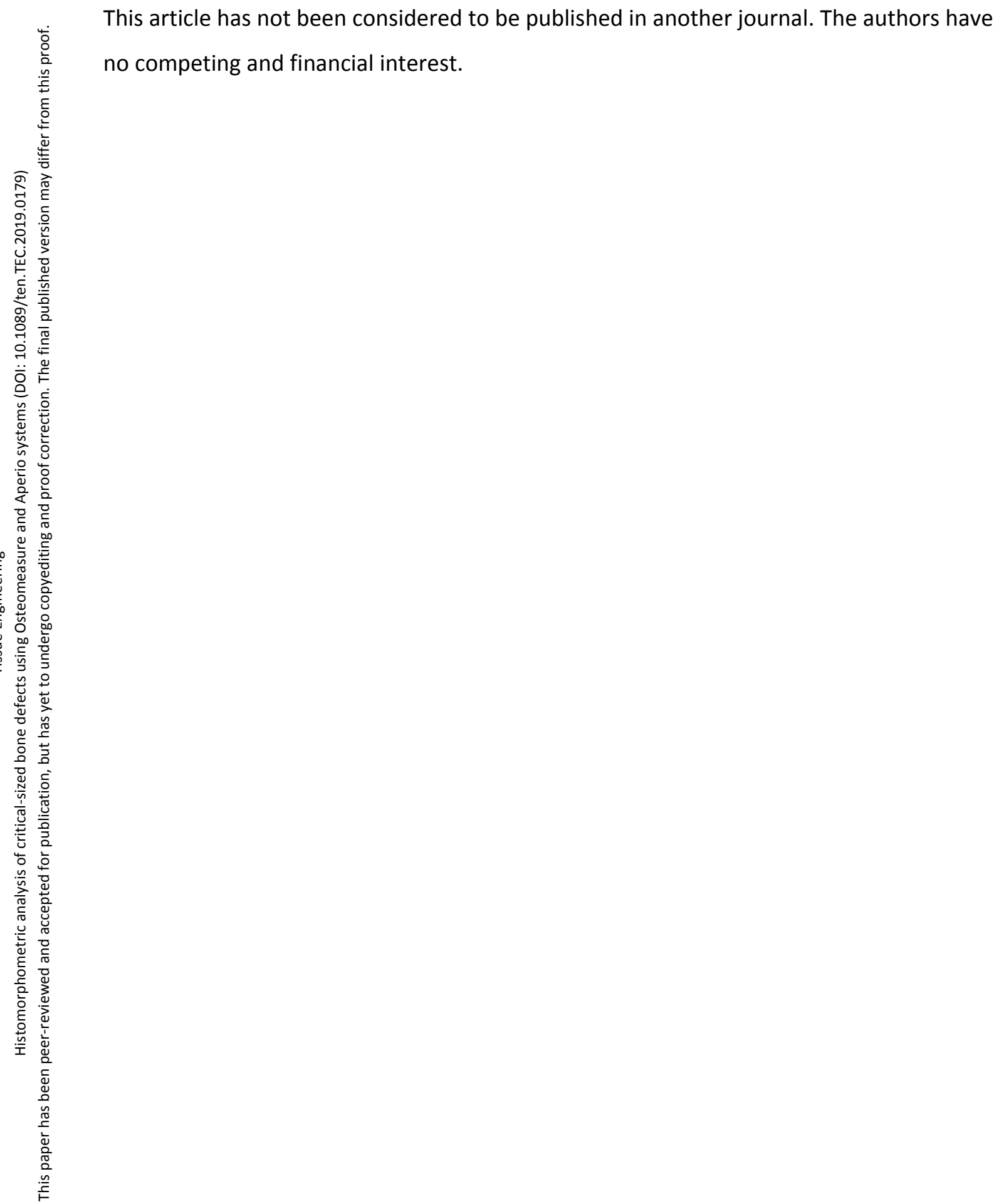




\section{References}

1. Hull P. The management of open tibial fractures. Eur J Orthop Surg Traumatol. 18, 441, 2008.

2. Marsell R, Einhorn TA. The biology of fracture healing. Injury. 42, 551, 2011.

3. Weibel ER. Measuring through the microscope: Development and evolution of stereological methods. J Microsc. 155, 393, 1989.

4. Zhang L, Chang M, Beck C a, et al. Analysis of new bone, cartilage, and fibrosis tissue in healing murine allografts using whole slide imaging and a new automated histomorphometric algorithm. Bone Res. 4, 15037, 2016.

5. Rentsch C, Schneiders W, Manthey S, et al. Comprehensive histological evaluation of bone implants. Biomatter. 4, e27993-1, 2014.

6. Lucaciu O, Gheban D, Soriţau O, et al. Comparative assessment of bone regeneration by histometry and a histological scoring system. Rev Rom Med Lab. 23, 31, 2015.

7. Fedchenko N, Reifenrath J. Different approaches for interpretation and reporting of immunohistochemistry analysis results in the bone tissue - a review. Diagn Pathol. 9, 221, 2014.

8. Collin P, Nefussi J, Wetterwald A, et al. Expression of Collagen, Osteocalcin, and Bone Alkaline-Phosphatase in a Mineralizing Rat Osteoblastic Cell-Culture. Calcif Tissue Int. 50, 175, 1992.

9. Detre S, Saccani Jotti G, Dowsett M. A 'quickscore' method for immunohistochemical semiquantification: validation for oestrogen receptor in breast carcinomas. J Clin Pathol. 48, 876, 1995.

10. Gerstenfeld LC, Wronski TJ, Hollinger JO, et al. Application of Histomorphometric Methods to the Study of Bone Repair. J Bone Miner Res. 20, 1715, 2005. 
11. Sojo K, Sawaki $\mathrm{Y}$, Hattori $\mathrm{H}$, et al. Immunohistochemical study of vascular endothelial growth factor (VEGF) and bone morphogenetic protein-2, -4 (BMP-2, -4) on lengthened rat femurs. J Cranio-Maxillofacial Surg. 33, 238, 2005.

12. Park SY, Kim KH, Koo KT, et al. The evaluation of the correlation between histomorphometric analysis and micro-computed tomography analysis in AdBMP-2 induced bone regeneration in rat calvarial defects. J Periodontal Implant Sci. 41, 218, 2005.

13. Rizzardi AE, Johnson AT, Vogel RI, et al. Quantitative comparison of immunohistochemical staining measured by digital image analysis versus pathologist visual scoring. Diagn Pathol. 7, 1, 2012.

14. Savi FM, Brierly GI, Baldwin J, et al. Comparison of Different Decalcification Methods Using Rat Mandibles as a Model. J Histochem Cytochem. 65, 705, 2017.

15. Jackson N, Assad M, Vollmer D, et al. Histopathological Evaluation of Orthopedic Medical Devices: The State-of-the-art in Animal Models, Imaging, and Histomorphometry Techniques. Toxicol Pathol. 47, 280, 2019.

16. Oryan A, Somayeh M, Bigham-Sadegh A. Bone Injury and Fracture Healing Biology. Biomed Environ Sci. 28, 57, 2015.

17. Kulak C a M, Dempster DW. Bone histomorphometry: a concise review for endocrinologists and clinicians. Arq Bras Endocrinol Metabol. 54, 87, 2010.

18. Rojo MG, Bueno G, Slodkowska J. Review of imaging solutions for integrated quantitative immunohistochemistry in the Pathology daily practice. Folia Histochem Cytobiol. 47, 349, 2009.

19. Deroulers $C$, Ameisen D, Badoual $M$, et al. Analyzing huge pathology images with open source software. Diagn Pathol. 8, 92, 2013.

20. Lejeune $M$, Jaén J, Pons $L$, et al. Quantification of diverse subcellular immunohistochemical markers with clinicobiological relevancies: Validation of a new computer-assisted image analysis procedure. J Anat. 212, 868, 2008. 
21. Pantanowitz L. Digital images and the future of digital pathology. J Pathol Inform. 1, 15, 2010.

22. Bancroft JD, Gamble M. Theory and practice of histological techniques. Philadelphia, PA : Churchill Livingstone/Elsevier, 2008.

23. Dempster DW, Compston JE, Drezner MK, et al. Standardized nomenclature, symbols, and units for bone histomorphometry: A 2012 update of the report of the ASBMR Histomorphometry Nomenclature Committee. J Bone Miner Res. 28, 2, 2013.

24. Gerstenfeld LC, Wronski TJ, Hollinger JO, et al. Application of histomorphometric methods to the study of bone repair. J Bone Miner Res. 20, 1715, 2005.

25. Parfitt AM, Drezner MK, Glorieux FH, et al. Bone histomorphometry: Standardization of nomenclature, symbols, and units: Report of the asbmr histomorphometry nomenclature committee. J Bone Miner Res. 2, 595, 1987.

26. Schlickewei CW, Laaff G, Andresen A, et al. Bone augmentation using a new injectable bone graft substitute by combining calcium phosphate and bisphosphonate as composite-an animal model. J Orthop Surg Res. 10, 1, 2015.

27. Glatt V, Canalis E, Stadmeyer L, et al. Age-related changes in trabecular architecture differ in female and male C57BL/6J mice. J Bone Miner Res. 22, 1197, 2007.

28. Sambrook PN, Hughes DR, Nelson AE, et al. Osteocyte viability with glucocorticoid treatment: Relation to histomorphometry. Ann Rheum Dis. 62, 1215, 2003.

29. Liu J, Li X, Zhang D, et al. Acceleration of Bone Defect Healing and Regeneration by Low-Intensity Ultrasound Radiation Force in a Rat Tibial Model. Ultrasound Med Biol. 44, 2646, 2018.

30. Attia A. Evidence-based medicine corner: Why should researchers report the confidence interval in modern research? Middle East Fertil Soc. 10, 78, 2005. 
31. Pantanowitz L, Farahani N, Parwani A. Whole slide imaging in pathology: advantages, limitations, and emerging perspectives. Pathol Lab Med Int. 7, 23, 2015.

32. Hyun Hong S. Computer-Automated Static, Dynamic and Cellular Bone Histomorphometry. J Tissue Sci Eng. 05, 1, 2012.

33. Cromey DW. Avoiding Twisted Pixels: Ethical Guidelines for the Appropriate Use and Manipulation of Scientific Digital Images. Sci Eng Ethics. 16, 639, 2010.

34. Hangartner TN. Thresholding technique for accurate analysis of density and geometry in QCT, pQCT and $\mu \mathrm{CT}$ images. J Musculoskeletal Neuronal Interac. 7, 9, 2007. 
Table 1. Inter system descriptive analyses of bone and fibrous tissue formation according to image analysis system, segmentation modes and variables ${ }^{1}$.

\begin{tabular}{|c|c|c|c|c|}
\hline \multirow[t]{2}{*}{$\begin{array}{l}\text { Descriptive } \\
\text { analysis }\end{array}$} & \multicolumn{2}{|c|}{ Osteomeasure x Aperio } & \multicolumn{2}{|c|}{ Osteomeasure x Aperio } \\
\hline & Automated & Automated & Automated & Automated \\
\hline Mean & 231.74 & 246.38 & 102.73 & 169.36 \\
\hline SD & 83.67 & 79.93 & 84.25 & 83.56 \\
\hline SEM & 20.29 & 19.38 & 20.43 & 20.27 \\
\hline Count & 17 & 17 & 17 & 17 \\
\hline $95 \% \mathrm{Cl}$ & $\begin{array}{l}191.96- \\
271.52\end{array}$ & $\begin{array}{l}208.37- \\
284.37\end{array}$ & $62.69-142.78$ & $\begin{array}{l}129.64- \\
209.08\end{array}$ \\
\hline \multirow{3}{*}{$\begin{array}{l}\text { Descriptive } \\
\text { analysis }\end{array}$} & \multicolumn{2}{|c|}{ Bone formation } & \multicolumn{2}{|c|}{ Fibrous tissue } \\
\hline & \multicolumn{2}{|c|}{ Osteomeasure x Aperio } & \multicolumn{2}{|c|}{ Osteomeasure $\mathrm{x}$ Aperio } \\
\hline & Manual & Manual & Manual & Manual \\
\hline Mean & 257.31 & 228.10 & 94.90 & 107.72 \\
\hline SD & 90.63 & 85.58 & 83.24 & 86.22 \\
\hline SEM & 21.98 & 20.76 & 20.19 & 20.91 \\
\hline Count & 17 & 17 & 17 & 17 \\
\hline $95 \% \mathrm{Cl}$ & $\begin{array}{l}214.23- \\
300.39\end{array}$ & $\begin{array}{l}187.42- \\
268.78\end{array}$ & $55.33-134.47$ & $\begin{array}{l}66.74- \\
148.71\end{array}$ \\
\hline \multirow{3}{*}{$\begin{array}{l}\text { Descriptive } \\
\text { analysis }\end{array}$} & \multicolumn{2}{|c|}{ Bone formation } & \multicolumn{2}{|c|}{ Fibrous tissue } \\
\hline & Osteomeasure & Aperio & Osteomeasure & Aperio \\
\hline & Automated & Manual & Automated & Manual \\
\hline Mean & 231.74 & 228.10 & 102.73 & 107.72 \\
\hline SD & 83.67 & 85.58 & 84.25 & 86.22 \\
\hline SEM & 20.29 & 20.76 & 20.43 & 20.91 \\
\hline Count & 17 & 17 & 17 & 17 \\
\hline
\end{tabular}

\footnotetext{
${ }^{1}$ SD (Standard deviation); SEM (Standard error of the mean); 95\% Cl (95\% coefficient interval).
} 
Page 22 of 45

22

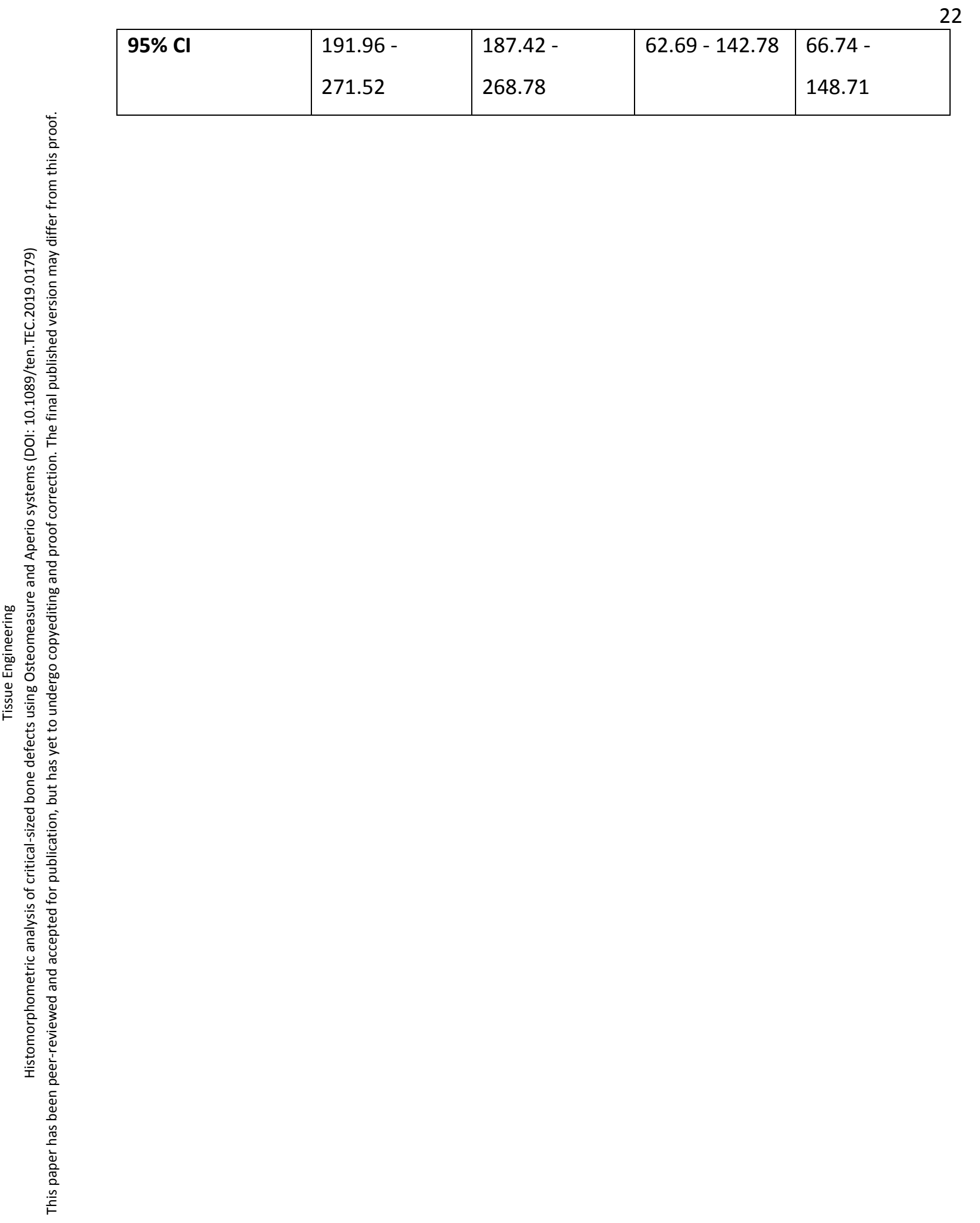


Table 2. Inter system descriptive analyses of bone and fibrous tissue formation per group treatment ${ }^{2}$.

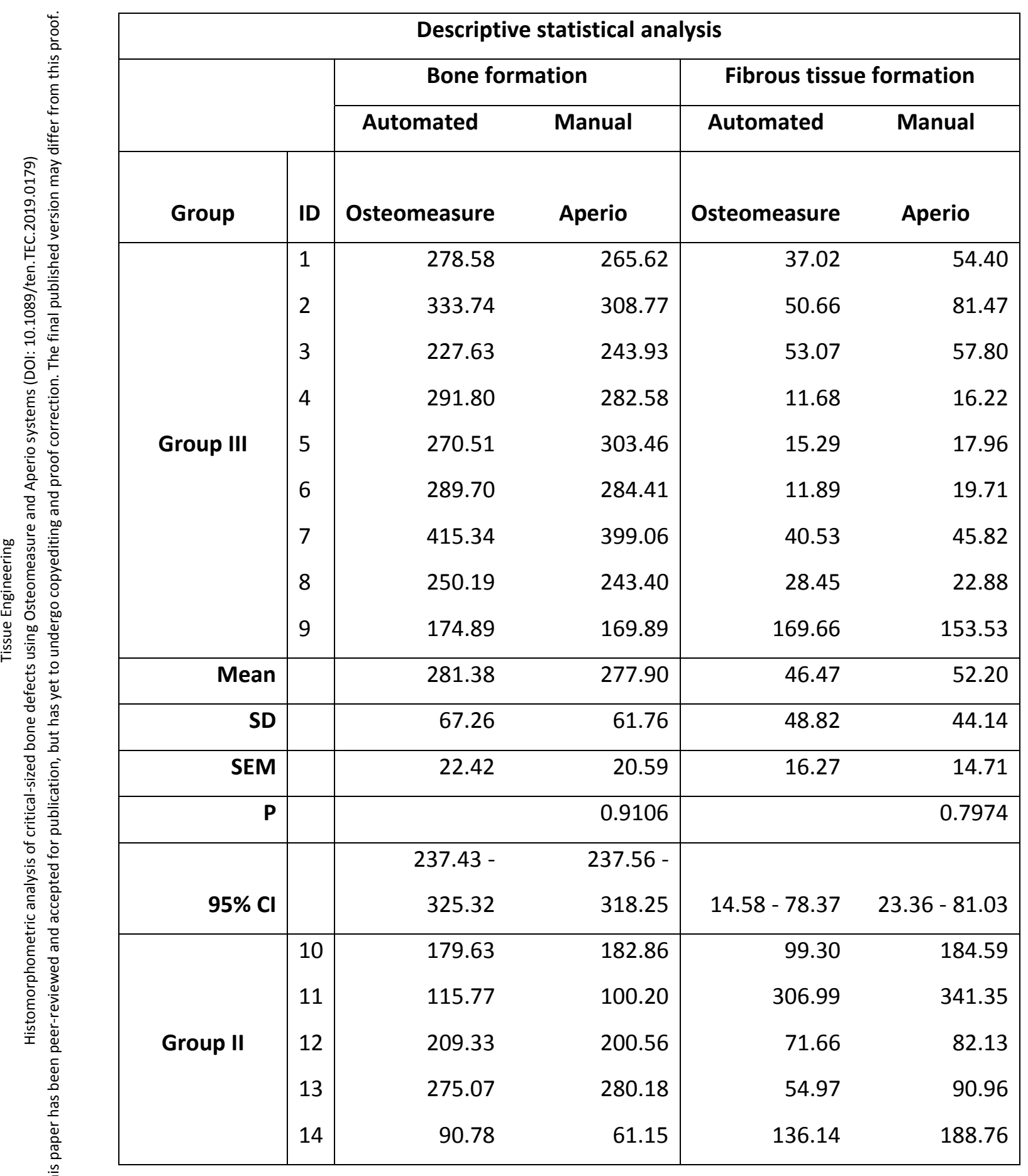

${ }^{2}$ SD (Standard deviation); SEM (Standard error of the mean); $p$ (probability); $95 \% \mathrm{Cl}$ (95\% coefficient interval). Group III (Microfiber mesh + alginate + BMP2); Group II (Microfiber mesh + alginate) and Group I (Microfiber mesh only). 
Page 24 of 45

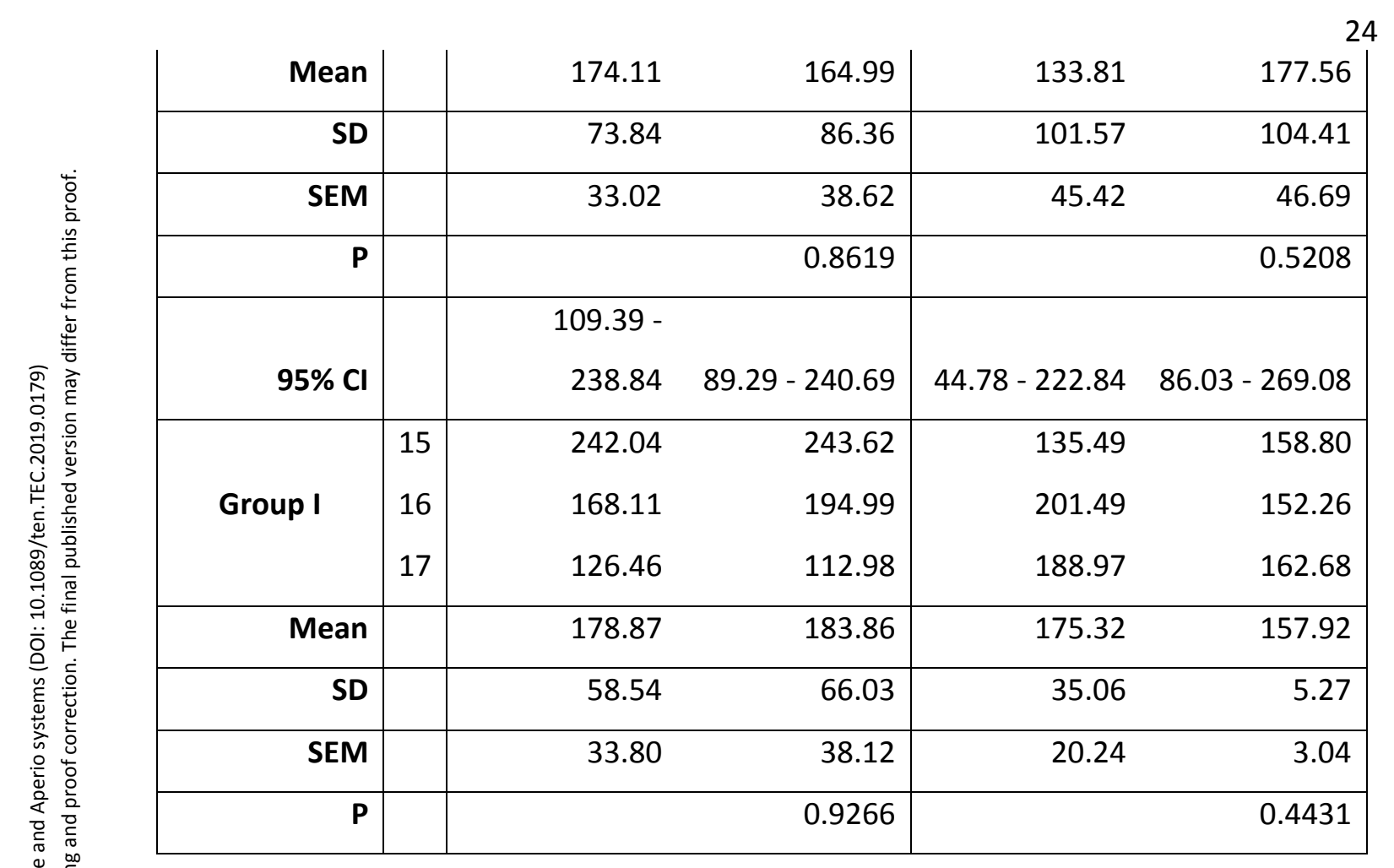


Figure legends

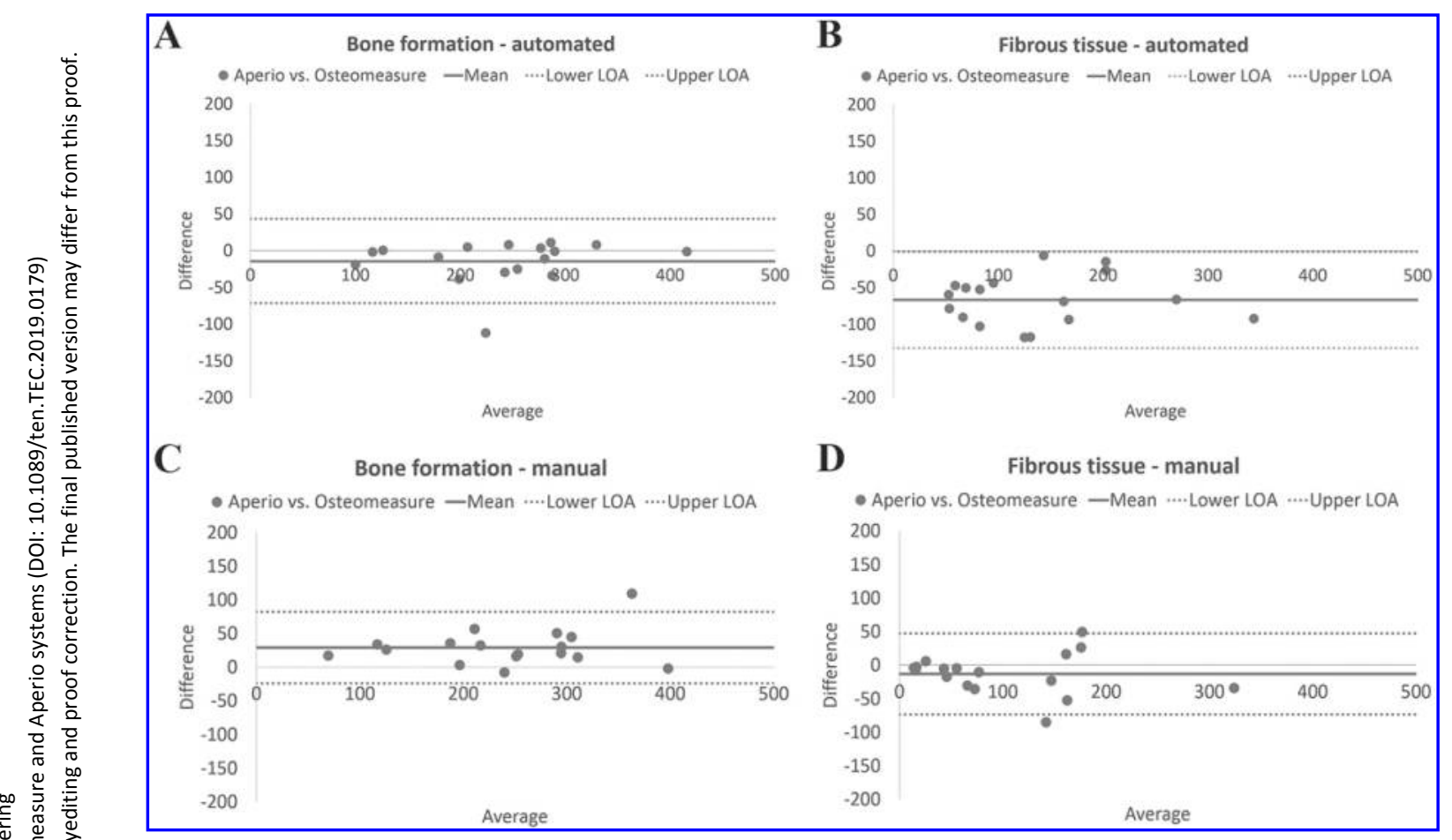

Figure 1. Inter system comparison between automated and manual segmentation modes using Osteomeasure and Aperio Image Scope systems for new bone and fibrous tissue formation variable. A) Bland-Altman plot comparing the Osteomeasure and Aperio Image Scope automated segmentation mode measurements of new bone formation histomorphometric variable, $\left(R^{2}=0.8754\right)$; B) Bland-Altman plot comparing the Osteomeasure and Aperio Image Scope automated segmentation mode measurements of fibrous tissue formation histomorphometric variable, $\left(R^{2}=0.8464\right)$; C) Bland-Altman plot comparing the Osteomeasure and Aperio Image Scope manual segmentation mode measurements of new bone formation histomorphometric variable, $\left.\left(R^{2}=0.9117\right) ; D\right)$ Bland-Altman plot comparing the Osteomeasure and Aperio Image Scope manual segmentation mode measurements of fibrous tissue formation histomorphometric variable, $\left(R^{2}=0.8734\right)$. 


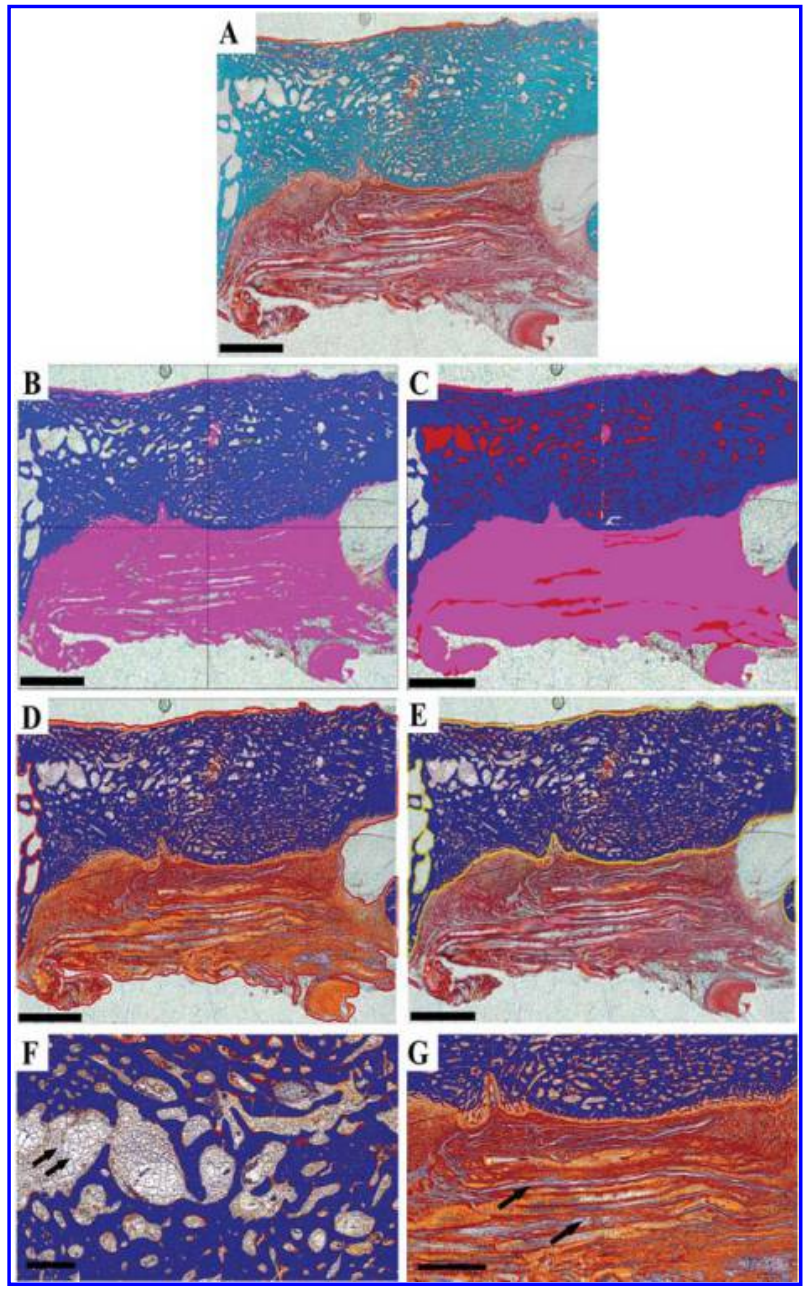

Figure 2. Comparison of Osteomeasure and Aperio Image Scope systems manual and automated segmentation modes. A) Bone defect Goldner's trichrome image overview (2.5X magnification); B) Osteomeasure system automated segmentation mode; C) Osteomeasure system manual segmentation mode; D) Aperio Image Scope automated segmentation mode; E) Aperio Image Scope system manual segmentation mode; F) and G) The images show that Aperio Image Scope system did not separate the background pixel colour in the void spaces within the void areas (background) in the bone (F) and fibrous tissues (G) areas (Black arrows: background within void spaces). Scale bar: A - E) 5 mm; F) 1 $\mathrm{mm}$ and G) $3 \mathrm{~mm}$. 


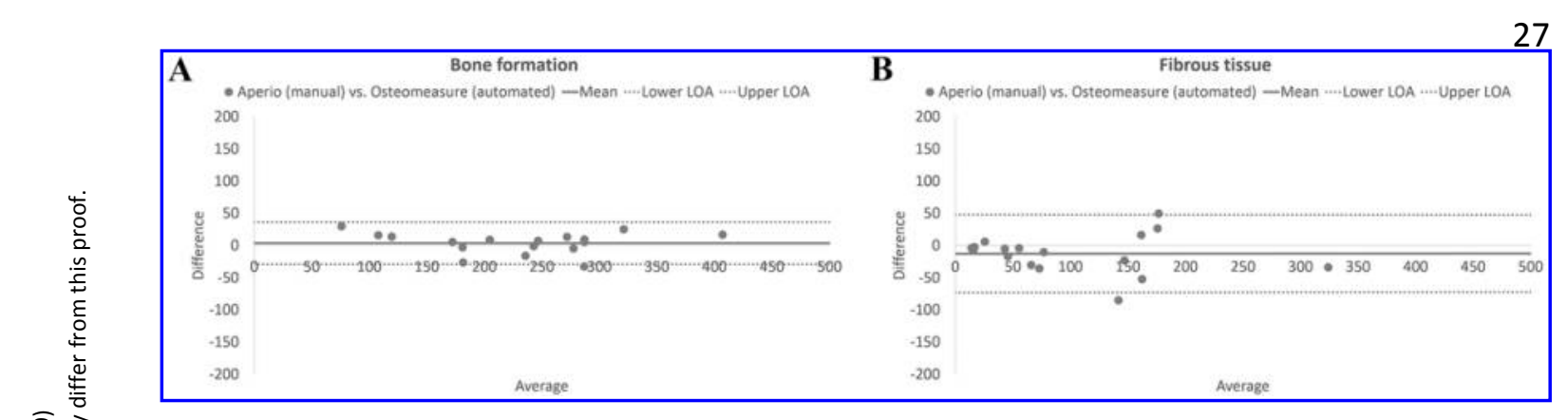

Figure 3. Inter system comparison between automated segmentation mode using Osteomeasure and manual segmentation mode using Aperio Image Scope systems, for new bone and fibrous tissue formation variables. A) Bland-Altman plot comparing the Osteomeasure automated segmentation mode measurements with manual Aperio Image Scope measurements of the bone formation histomorphometric variable, $\left.\left(R^{2}=0.9615\right) ; B\right)$ Bland-Altman plot comparing the Osteomeasure automated segmentation mode measurements with manual Aperio Image Scope measurements of the fibrous tissue formation histomorphometric variable; $\left(R^{2}=0.8734\right)$. 


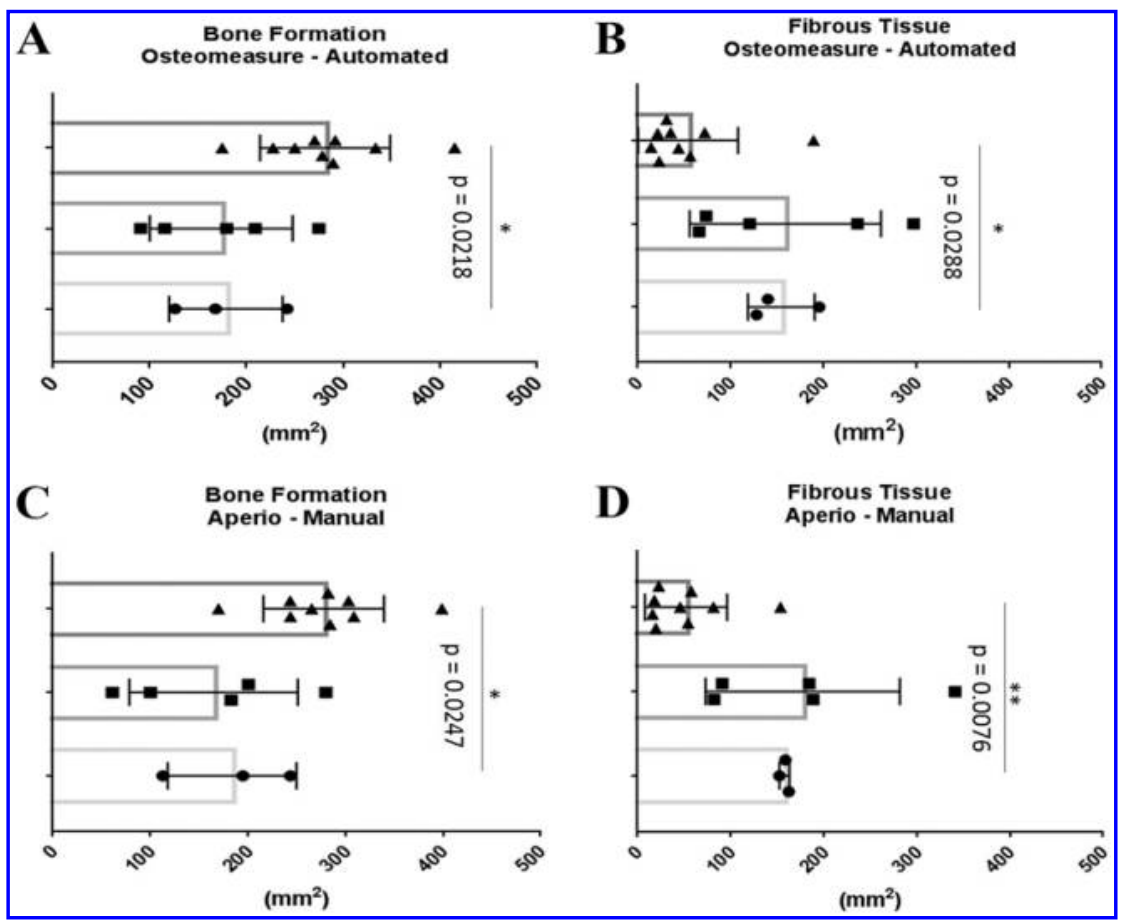

Figure 4. Anova analysis for new Bone and Fibrous tissue formation. A) New bone formation using the Osteomeasure automated segmentation mode ( $p=0.0218) ; B)$ Fibrous tissue formation using the Osteomeasure automated segmentation mode $(p=$ 0.0288); C) New bone formation using the Aperio Image Scope manual segmentation mode $(p=0.0247)$ and D) Fibrous tissue formation using the Aperio Image Scope manual segmentation mode $(p=0.0076)$. 


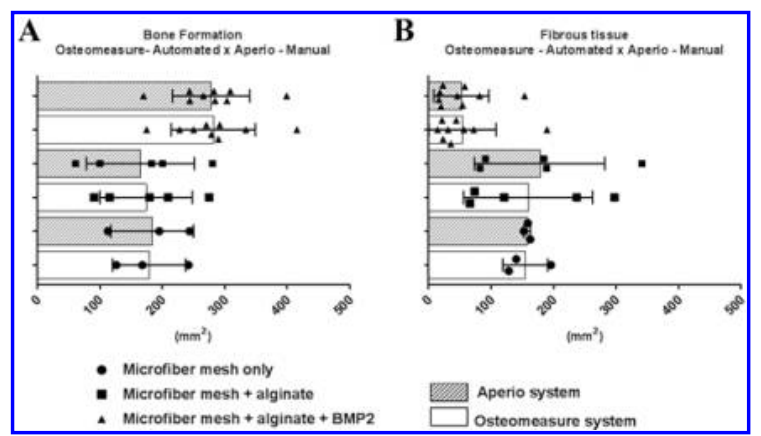

Figure 5. Comparison of the measurements trend obtained with the automated segmentation mode with the Osteomeasure system and with the manual segmentation mode with the Aperio Image Scope image analysis systems. A) Bone formation histomorphometric variable and B) Fibrous tissue formation histomorphometric variable. 


\section{Supplemental material 1: Histological sample preparation}

Three different treatment groups, each containing samples with a critical sized $3 \mathrm{~cm}$ tibial defect, were evaluated in this study: In group I $(n=3)$, a microfiber mesh-scaffold only was implanted into the empty defect site. In group II $(n=5)$, the microfiber mesh-scaffold was combined with $6 \mathrm{ml}$ of functionalized (with Arginine, Glycine, Aspartate (RGD) peptide sequences) alginate hydrogel injected into the scaffold lumen after implantation. In group III ( $\mathrm{n}=9), 6 \mathrm{ml}$ of functionalized alginate hydrogel combined with $2 \mathrm{mg}$ recombinant human bone morphogenic protein 2 (rhBMP - 2) were injected into the scaffold lumen after implantation.

All animals were euthanized 6 months post-surgery. Sheep tibia were dissected from the surrounding tissue and fixed in $10 \%$ formaldehyde (Sigma-Aldrich, Castle Hill, Australia) for 1 week. After fixation, the tibias were cut to $50 \mathrm{~mm}$ length $(30 \mathrm{~mm}$ defect length plus $1 \mathrm{~cm}$ of proximal and $10 \mathrm{~mm}$ distal host bone). Two sagittal sections (3-5 $\mathrm{mm}$ thick) were collected from each sample using an EXAKT 310 Diamond Band Saw (EXAKT Apparatebau GmbH \& Co.KG, Norderstedt, Germany). The samples were processed and resin embedded in the low-temperature embedding system Technovit $9100 \mathrm{New}^{\circledast}$ (Heraeus Kulzer GmbH, Germany) following the protocol describe by Willbold \& Witte. ${ }^{34}$ Resin blocks were mounted, sectioned and ground to $50 \mu \mathrm{m}$ using an EXAKT 400CS micro grinder (EXAKT Apparatebau GmbH \& Co.KG, Norderstedt, Germany) according to the technique described by Donath. ${ }^{35}$

Resin sections were etched using one change of xylene and one change of $100 \%$ ethanol for 30 minutes each and subsequently hydrated in two changes of ethanol from $90 \%$ to $70 \%$, followed by one change in distilled (DI) water for 5 minutes each. Slides were stained with Weigert's hematoxylin solution for 25 minutes, Ponceau-fuchsin solution for 10 minutes; Orange $\mathrm{G}$ solution for 20 minutes and Light Green solution for 45 minutes. Sections were dehydrated in two changes of ethanol (70\% and 100\%) for 30 seconds each, then dried overnight and coverslipped using EUKIT mounting medium. 


\section{Supplemental material 2: Image acquisition and Image properties}

\section{Image acquisition}

Exposure, white balance and shade correction were performed. Following this, using the brightfield tiles option, the border areas of the tissue samples were defined and tiled images were acquired. The tiles were assembled (stitched together) into a whole image and exported through the ZEN software program in TIFF format to be used in the Osteomeasure system and in Big TIFF pyramid format to be used in the Aperio Image Scope system.

A sub-set of images limited to the defect site only was defined using the Aperio Image Scope image analysis system. Using the ruler tool, the defect perimeter of $30 \mathrm{~mm}$ was defined excluding proximal and distal host bone. The defect only ROls consistently comprised 11623 pixels $(30 \mathrm{~mm}$ ) in length, which was defined in the settings of the extract image region option of the Aperio Image Scope image analysis system. Height was not standardized as the width of each defect was not consistent, ranging from 7242 to 10003 pixels (average: 8918 pixels, $23 \mathrm{~mm}$ ). Images of the defect only ROI were used for all histomorphometric evaluation

\section{Image properties}

The manner in which a digital image is displayed is dependent on the microscope conditions/parameters/properties used to acquire the image. ${ }^{36}$ To load and calibrate an image data set in the Osteomeasure system, both the image dimensions (length and height) in microns/pixels as well as the scaling factor $(2.58 \mu \mathrm{m} /$ pixel) were retrieved from the czi experiment file properties information (Fig. 1). To load and calibrate an image data set in the Aperio Image Scope, only the scaling factor $(2.58 \mu \mathrm{m} /$ pixel) needed to be retrieved from the czi experiment file properties information (Fig. 1). To load and calibrate an image data set in the Aperio Image Scope, only the scaling factor $(2.58 \mu \mathrm{m} / \mathrm{pixel})$ needed to be retrieved from the czi experiment file properties information (Fig. 1). 
Page 32 of 45

32

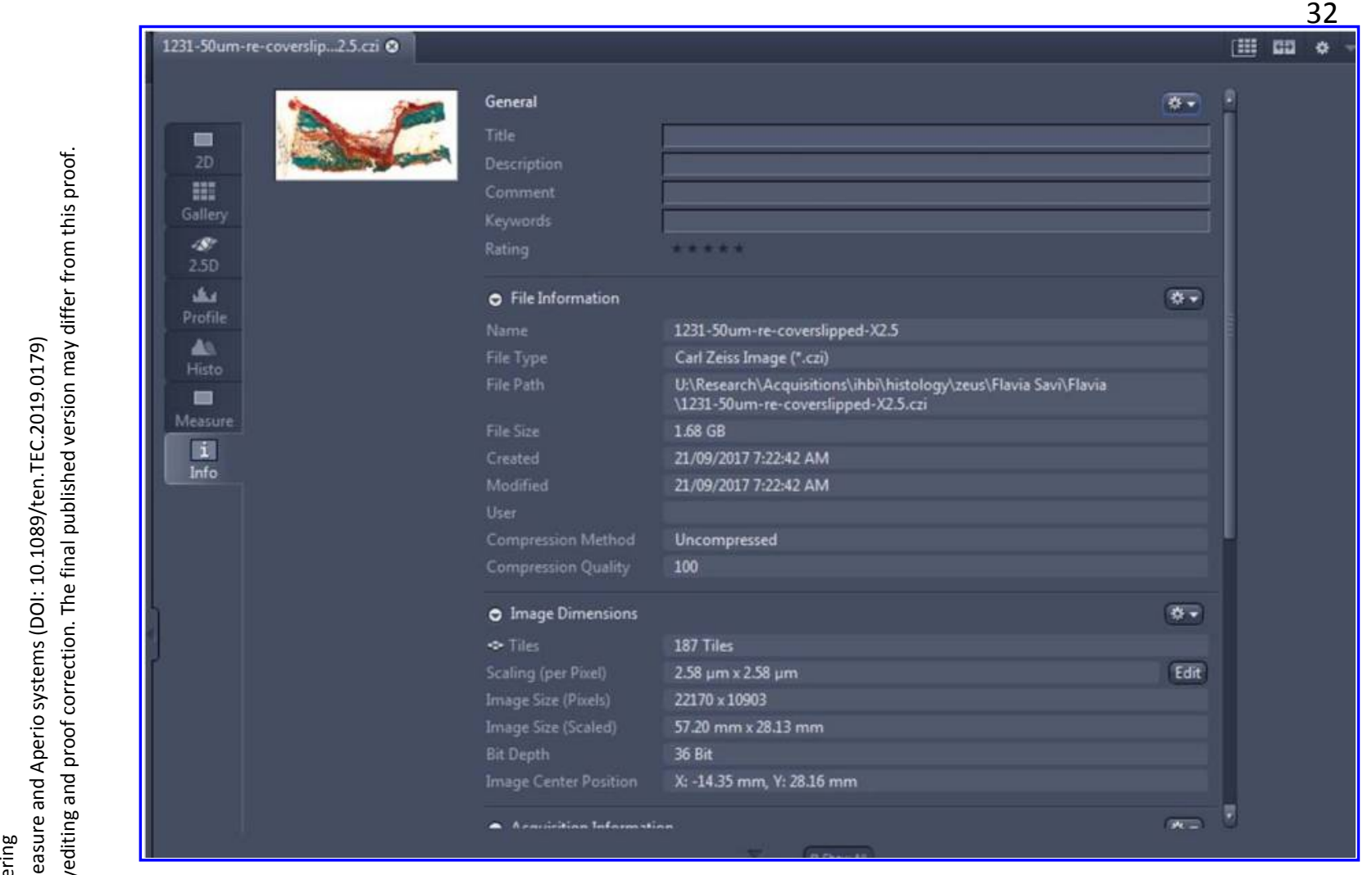

Supplementary Figure 1. CZI image properties file. Each image has file information related to the microscope parameters used for acquiring the image. These raw data include: image dimensions and scaling factor (microns/pixel). 


\section{Supplemental material 3: Histomorphometry - Osteomeasure}

To perform the histomorphometric evaluation, the image subset for each defect only ROI was segmented into separated fields of view (FOV) to (1) more accurately delineate the desired features and (2) better identify possible miscalculations during the image assessment. Using the $2 \mathrm{X}$ magnification setting in the Osteomeasure software, four separate FOV were assessed.

The histomorphometric variables were selected either manually by contouring around the tissue variables or automatically based on color patterns of the histological stain using thresholding. All pixels within the contour (manual) or color (automated) range were selected. A user-assigned, single referential color for each variable was displayed as a color mask in the forefront image. After all variables had been assessed the software accumulated individual fields data view with the primary data in each field view, as well as a data summary spreadsheet for the whole section with all variables measured. The system used $\mathrm{mm}^{2}$ unit for the area measurements.

\section{Osteomeasure protocol for static images using the manual mode.}

1. On main screen, select Osteomeasure software icon. (A red box appears on a black screen);

2. On the Osteomeasure menu bar select FILE BIOPSY;

3. Read TIFF file and select image file to be analysed;

4. To ensure CALIBRATION is appropriate, do the following;

CALIBRATION / SET BOX SIZE TO TIFF IMAGE (a message pops up asking: Do you want to proceed? Select YES;

5. From the menu bar select CALIBRATION / NEW CALIBRATION (A message appears indicating that: the current calibration will be overwritten. Do you want to save as another name: select NO to continue without saving. A Box appears with objective/zoom factors (This list of objectives is only needed when there is a microscope attached to the Osteomeasure system). Ignore this box as we are calibrating a static or saved image file. Select DONE.

6. Ignore the message that appears in the grey box; 
7. From the menu bar select CALIBRATION / CALIBRATION OFF

A box for $X / Y$ dimensions appears. For successful calibration, the user needs to enter the specific $X / Y$ dimensions in microns for the TIFF file currently being read. To determine these dimensions, retrieve the image dimensions in pixels from the properties tab and multiple them by the relevant scaling factor (e.g At $2 \mathrm{X}$ magnification, the scaling factor is 2.58 microns/pixel on the Zeiss $M 2$ images).

8. Type in the $X / Y$ dimensions in microns and save the new calibration;

\section{Before making measurements:}

9. To set tissue boundaries and customize bone variables go to:

10. OPTIONS / MISC / in "irregular tissue measurements": select DRAW TISSUE AREA Also in "summary display options": select CUSTOM and AUTO-CUSTOM.

Note: If the user does not select these options, measurements will not be recorded. Use tissue boundaries for posterior percentage (\%) calculations of the total area measured in a sample.

\section{When using manual mode.}

11. In OPTIONS / CUSTOM set specific parameters for normal area measurements (e.g. Soft tissue, Host bone) including selecting a user-assigned referential specific color; Note: Do not forget to tick the box for the specific measurements; if not ticked these measurements will not appear in the parameter list of the Osteomeasure main window.

12. In OPTIONS / AUTO-CUSTOM set specific parameters for auto areas measurements - "to be used with Threshold function" - by selecting specific color (e.g. Alginate); Note: Do not forget to tick the box for the specific measurements; if not ticked these measurements will not appear in the parameter list of the Osteomeasure main window.

13. In OPTIONS select MEASURE VIEW;

14. On the image control menu, increase magnification to $2 X$ (Top right $\uparrow \downarrow$ Objective); 
15. Because the option "DRAW TISSUE AREA" is selected, when the user starts to make measurements the system will ask: "Are you sure you would like to set irregular tissue border? Select Yes. 


\section{Supplemental material 4: Histomorphometry - Aperio Image Scope}

In conventional digital imaging, image color pixel information is provided in red, green and blue (RGB) color mode. In modern image analysis systems, such as the Aperio Image Scope image color pixel settings are based on the hue, saturation and intensity (HSI) color mode. This mode specifies the color using a color wheel spectrum (Fig. 2 C); each color is numerically represented from 0 to 1 . Saturation refers to the purity of the hue (0-1), and hue width relates to the hue color range (0-1), which means that in the wheel spectrum, the lower the hue width and saturation selection, the more restricted the pixel selection will be. Hue intensity is related to the brightness of the pixel and is represented by the mean of the red + green + blue pixel values, ranging from black (0) to white (255) and representing the intensity parameters for the weak (yellow color mask selection), medium (orange color mask selection) and strong positive (red color mask selection) pixel staining color selection within an image (Fig. 2 E) (Aperio Image Analysis User's Guide. Aperio Technologies Inc. CA, US. Available from: http://tmalab.jhmi.edu/aperiou/userguides/Image Analysis UG.pdf).

Therefore, analysis of histological data sets within Aperio Image Scope should be performed only following conversion of the RGB to HSI color mode. The protocol (Fig. 2 and Supplemental Material 2 and 3 ) for this conversion is as follows:

The pixel RGB colour value for each of the Goldner's trichrome stain colours was extracted using the MatLab program through the pixel information command (Fig. 2 A). To account for colour heterogeneity of the Goldner's trichrome stain, a range of RGB hue threshold values for each variable (bone and fibrous tissue) were selected from five ROIs within each image data set. The RGB pixel values were subsequently converted to an HSI mode. A hue value threshold of 0.16 was used in the algorithm's parameter input of the Aperio Image Scope algorithm (Fig. 2 B and D). After analyzing the defect only ROI images, a pseudo color image was generated with highlighted features of interest and aided visual interpretation of the results (Fig. $2 \mathrm{E}$ and table 1). The defect only results were assessed through the annotation menu tab of the Aperio Image Scope image analysis software. The results were evaluated according to staining intensity: Number of Weak Positive (NWP); 
Number of Positive (NP), Number of Strong Positive (NSP) and Number of Negative (NN) (Fig. 2 F). The NP and NSP were used for the fibrous tissue and the NN was used for the bone formation quantification. The measurements obtained for the total tissue area were accessed through the view/menu annotations tab and exported in .xls format for further statistical analysis (Fig. 2 F). The absolute pixel numbers obtained with Aperio Image Scope for bone and fibrous tissue variables were converted using the scaling factor to $\mathrm{mm}^{2}$. 


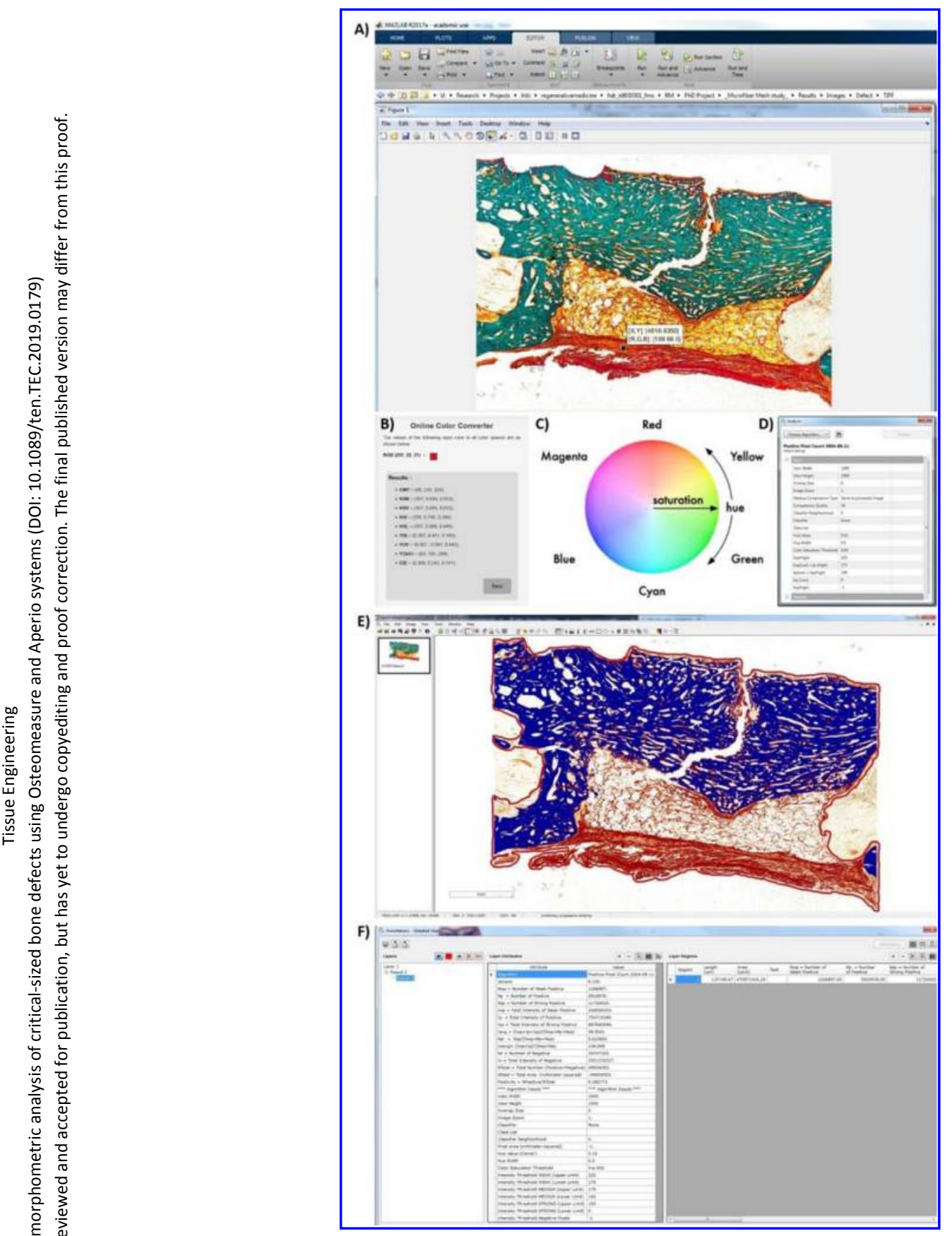

Supplementary Figure 2. Aperio Image Scope image analysis sequence. A) Obtaining RGB pixel values using MatLab; B) Converting RGB colour to HSI color mode (source: http://www.picturetopeople.org/color_converter.html); C) HSI color wheel spectrum (source: https://www. highend.com/pub/support/controllers/documents/HTML/en/sectcolour_matching.htm); D) Algorithm input settings; E) Aperio Image Scope algorithm color mask selection and F) Aperio Image Scope histomorphometric output. 


\section{Aperio Image Scope protocol for static images.}

1. Select Aperio Image scope software icon.

2. From the menu bar select FILE/OPEN IMAGE;

3. Select the image file to be analysed;

4. To ensure CALIBRATION is appropriate, on menu bar select IMAGE/RESOLUTION and type in the scaling factor for the image acquired;

5. With the pen tool, select the tissue boundaries to be analysed (manual mode); at this point the user can select one variable at time.

6. With the RECTANGLE TOOL (F5) the user can select the entire image view to be analysed (automated mode);

7. If required, select the negative pen tool to exclude unwanted areas such as image background from the image analysis.

8. After selecting the areas to be analysed go to the menu bar and select VIEW TAB/ANALYSIS (a box will pop on the screen). Then choose the algorithm POSITIVE PIXEL COUNT (PPC);

9. After selecting the PPC, the user can adjust hue value and hue width according to the staining color that is going to be analysed.

10. At this point the user must find out the RGB color value for a specific stained structure or area.

11. To obtain the color the user can use MatLab command: IMSHOW ('image file name');

12. The image will appear on the screen. Underneath the menu bar, select the DATA CURSOR icon and place it over the desired color. The RGB color values will appear.

13. Convert the RGB color values to HSI mode and divide by 360 . This calculation will give you the exact color to use in the PCC algorithm.

14. After typing the color into the algorithm input, select ANALYSE ANNOTATIONS.

15. A color mask selection will be generated and the user can evaluate if the selection made by the algorithm is satisfactory.

16. Once the user is happy with the selection made by the software, go to the menu bar/ VIEW TAB/ANNOTATIONS and EXPORT TO EXCEL the measurements obtained. 
17. The measurements obtained will be given in number of pixels, except for the image total area which is in $\mathrm{mm}^{2}$.

18. If the user wants to have all the measurements in $\mathrm{mm}^{2}$, then the user must calculate the measurements using the resolution or scaling factor obtained while acquiring the image file, which is given in microns per pixel. 
Supplemental material 5: Image data set and representative image segmentation mask selection

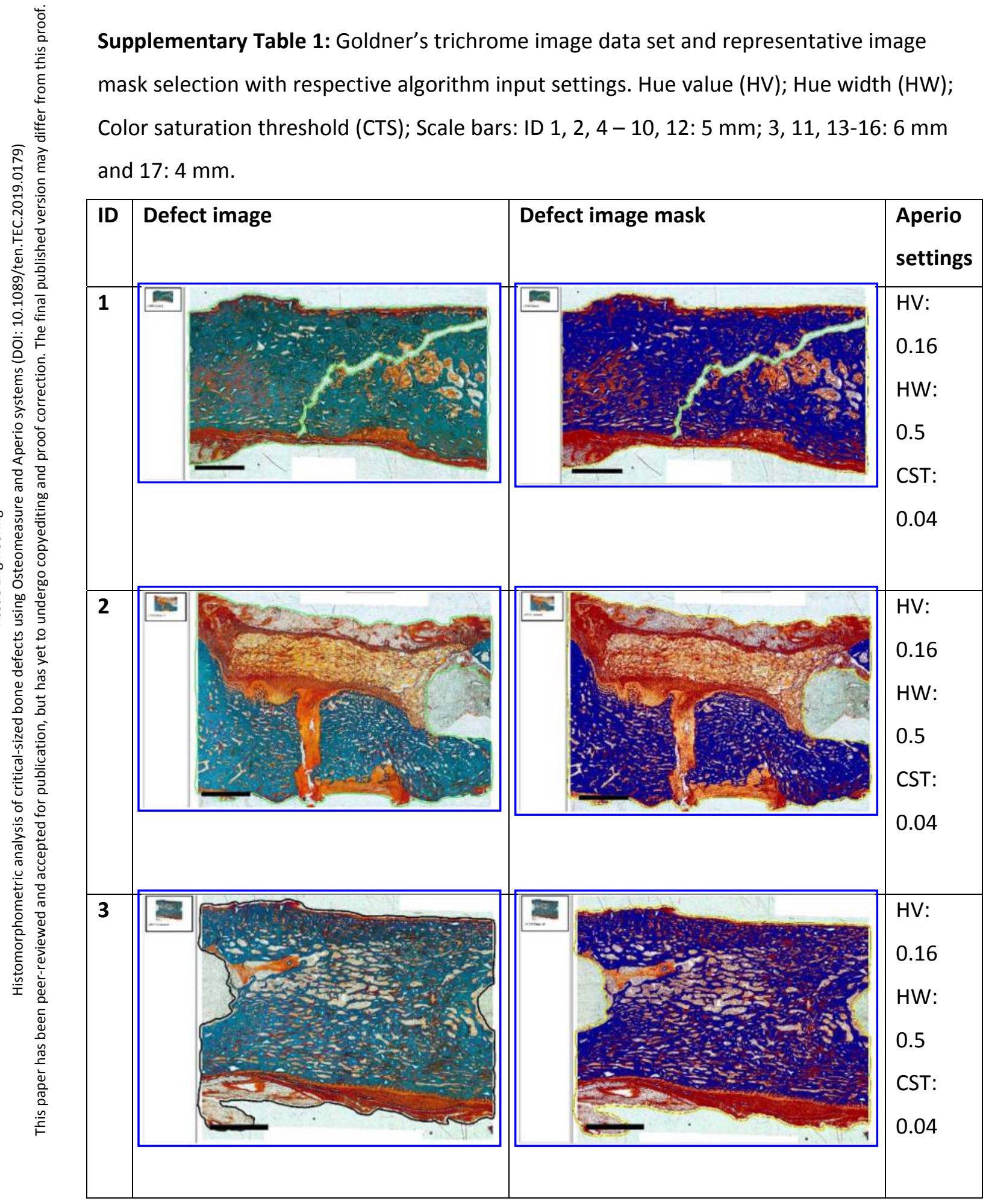




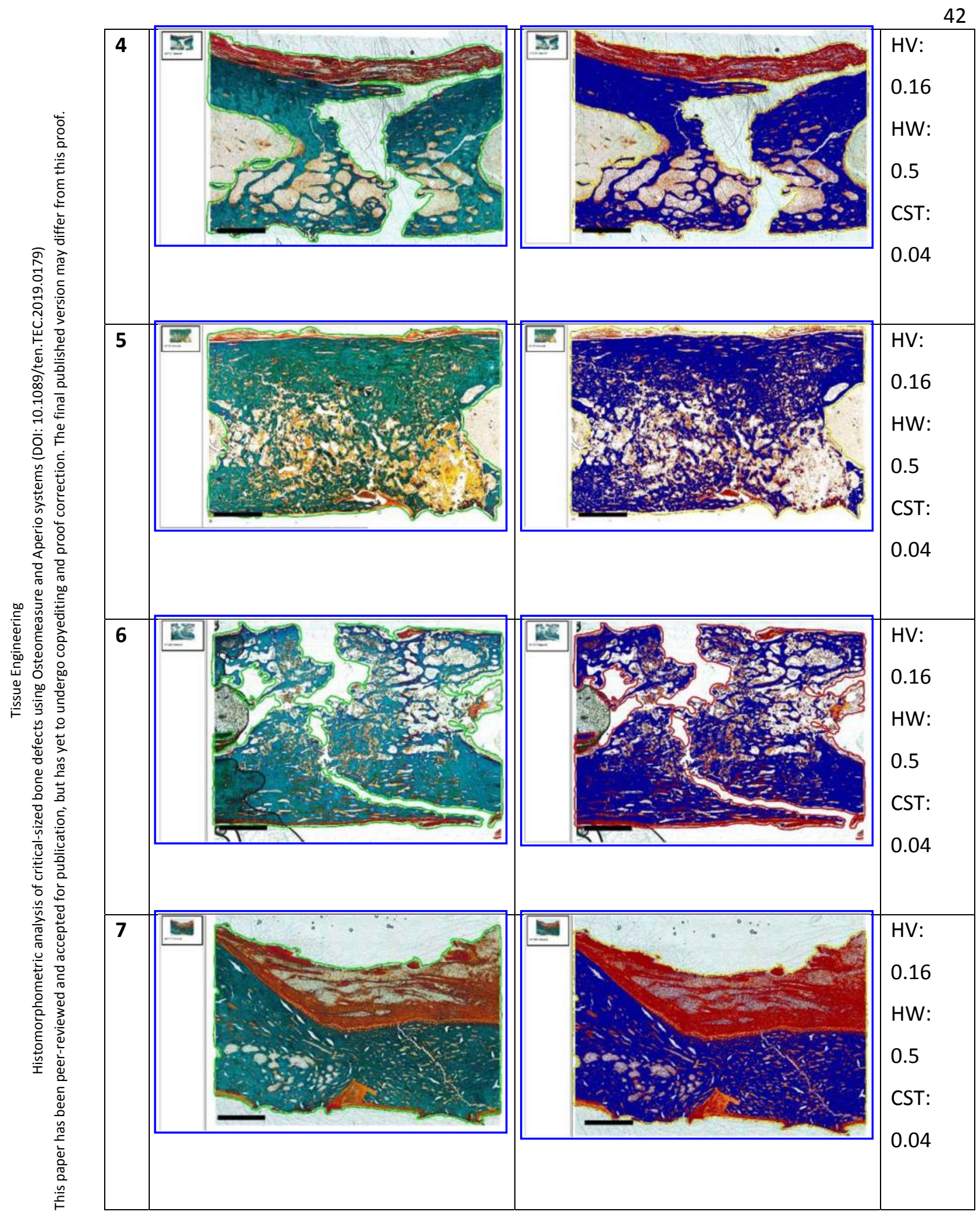




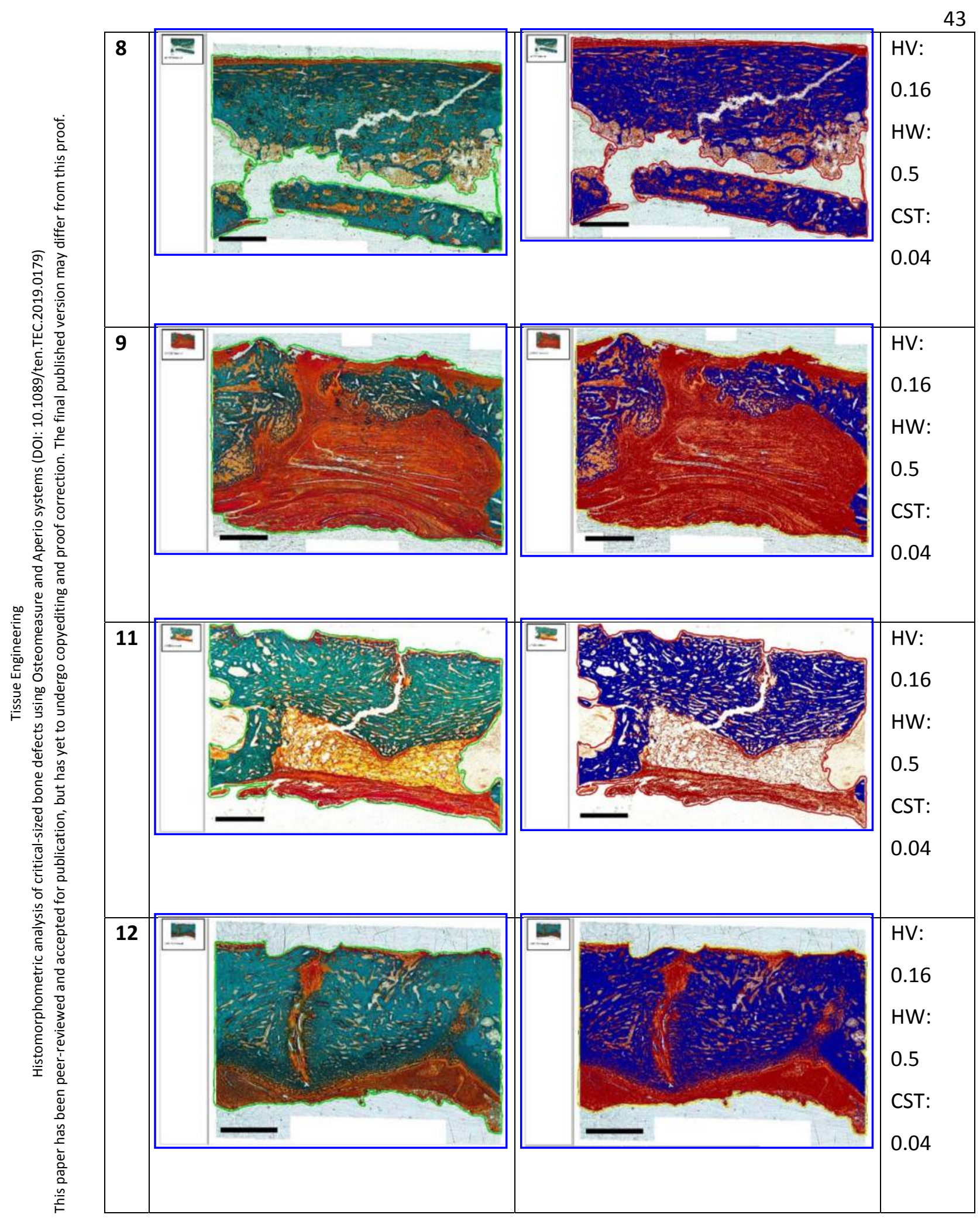




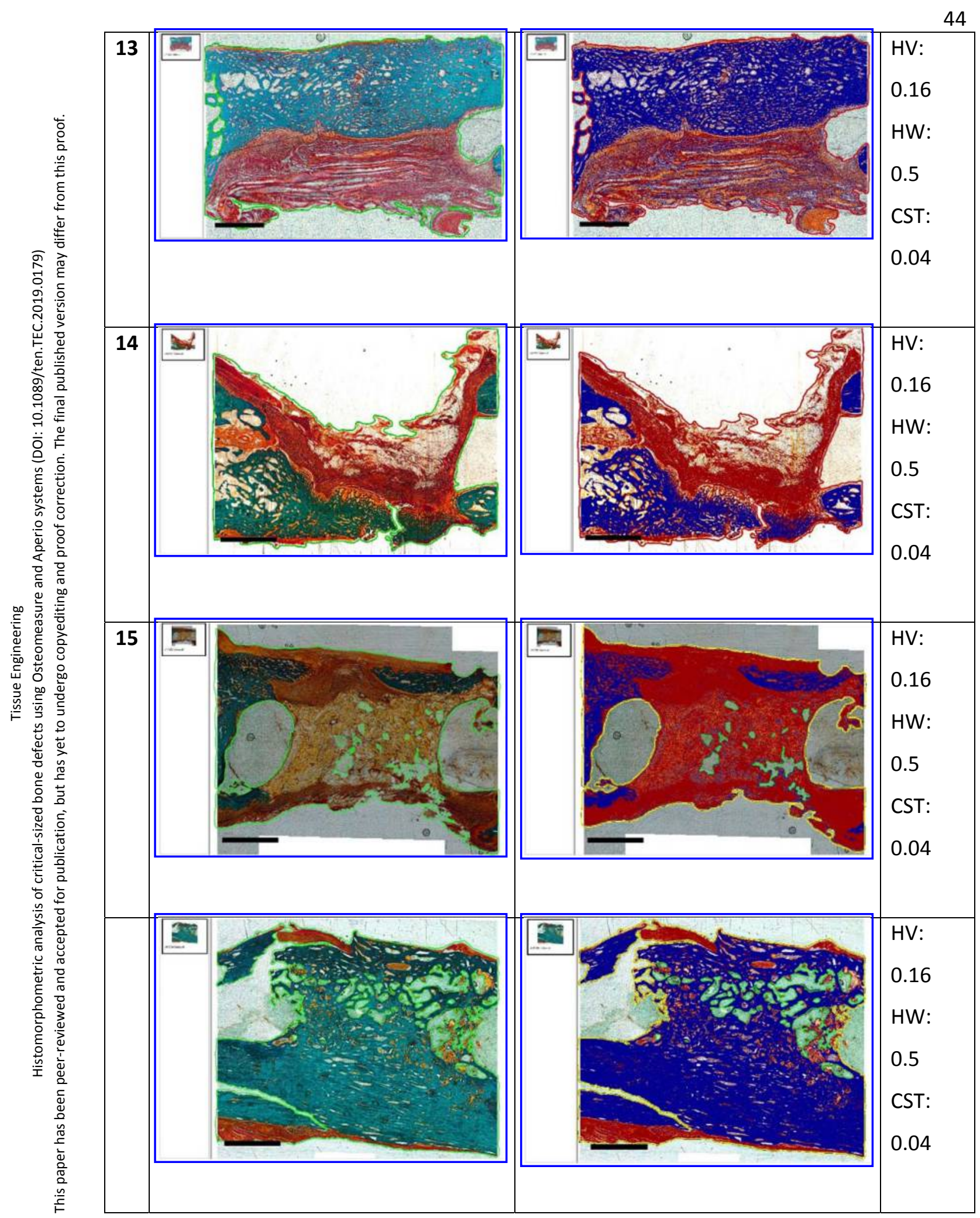




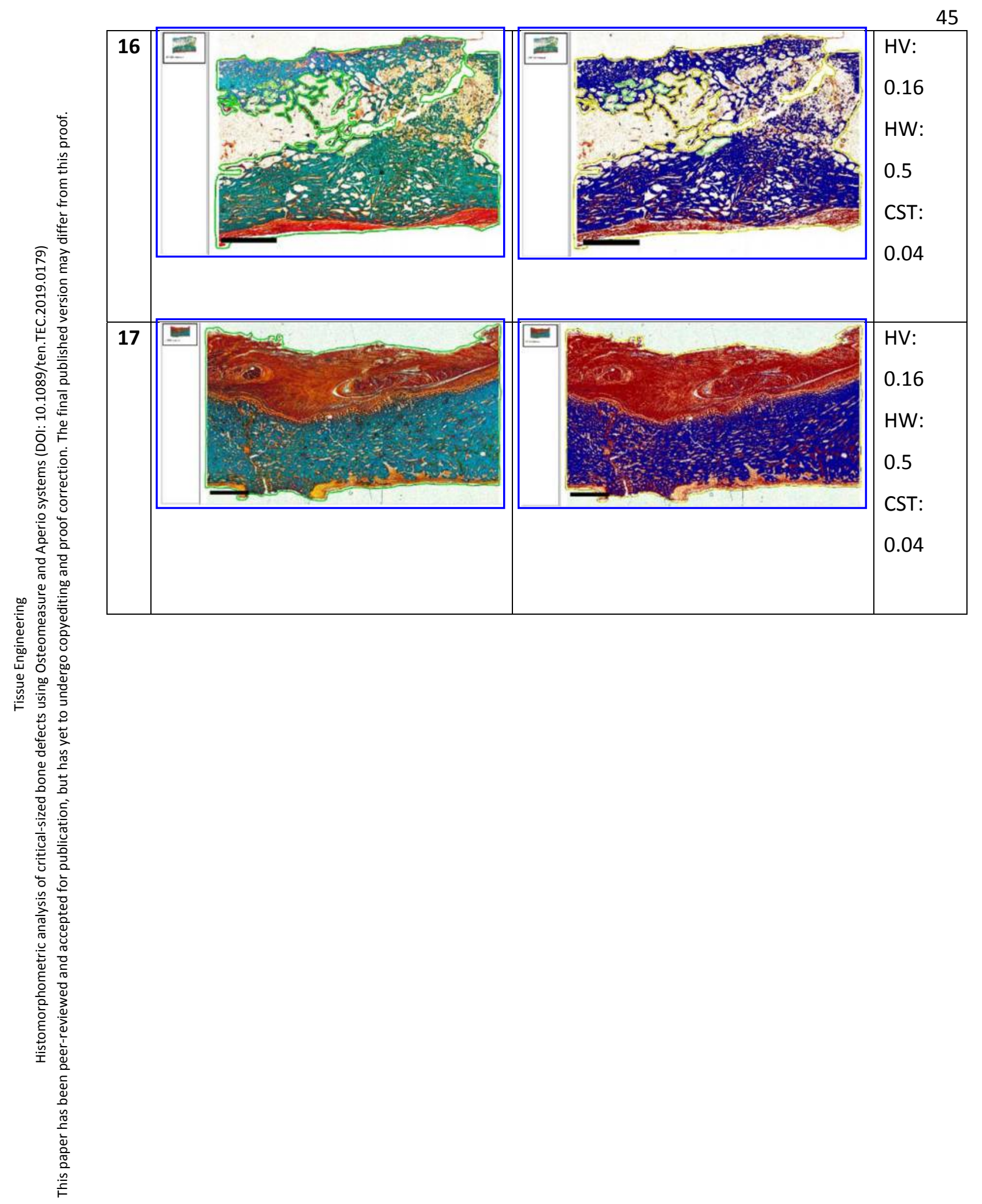

